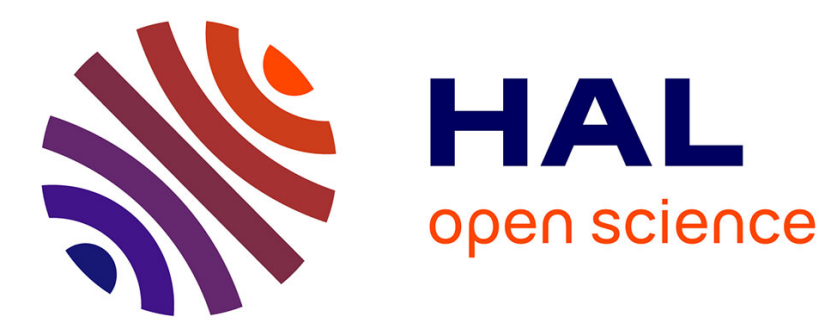

\title{
Non-diffusive resonant acceleration of electrons in the radiation belts
}

\author{
A. V. Artemyev, Vladimir Krasnoselskikh, O. V. Agapitov, Didier Mourenas, \\ G. Rolland
}

\section{- To cite this version:}

A. V. Artemyev, Vladimir Krasnoselskikh, O. V. Agapitov, Didier Mourenas, G. Rolland. Nondiffusive resonant acceleration of electrons in the radiation belts. Physics of Plasmas, 2012, 19 (12), pp.122901. 10.1063/1.4769726 . insu-01259507

\section{HAL Id: insu-01259507 https://hal-insu.archives-ouvertes.fr/insu-01259507}

Submitted on 20 Jan 2016

HAL is a multi-disciplinary open access archive for the deposit and dissemination of scientific research documents, whether they are published or not. The documents may come from teaching and research institutions in France or abroad, or from public or private research centers.
L'archive ouverte pluridisciplinaire HAL, est destinée au dépôt et à la diffusion de documents scientifiques de niveau recherche, publiés ou non, émanant des établissements d'enseignement et de recherche français ou étrangers, des laboratoires publics ou privés. 


\section{AIP | Physisos of}

\section{Non-diffusive resonant acceleration of electrons in the radiation belts}

A. V. Artemyev, V. V. Krasnoselskikh, O. V. Agapitov, D. Mourenas, and G. Rolland

Citation: Physics of Plasmas 19, 122901 (2012); doi: 10.1063/1.4769726

View online: http://dx.doi.org/10.1063/1.4769726

View Table of Contents: http://scitation.aip.org/content/aip/journal/pop/19/12?ver=pdfcov

Published by the AIP Publishing

\section{Articles you may be interested in}

Laboratory studies of nonlinear whistler wave processes in the Van Allen radiation belts

Phys. Plasmas 22, 091503 (2015); 10.1063/1.4928944

Nonlinear electron acceleration by oblique whistler waves: Landau resonance vs. cyclotron resonance Phys. Plasmas 20, 122901 (2013); 10.1063/1.4836595

Resonance zones and quasi-linear diffusion coefficients for radiation belt energetic electron interaction with oblique chorus waves in the Dungey magnetosphere

Phys. Plasmas 19, 072904 (2012); 10.1063/1.4737105

Refractive index and wavenumber properties for cyclotron resonant quasilinear diffusion by cold plasma waves

Phys. Plasmas 14, 072901 (2007); 10.1063/1.2744363

Computer simulations of relativistic whistler-mode wave-particle interactions

Phys. Plasmas 11, 3530 (2004); 10.1063/1.1757457

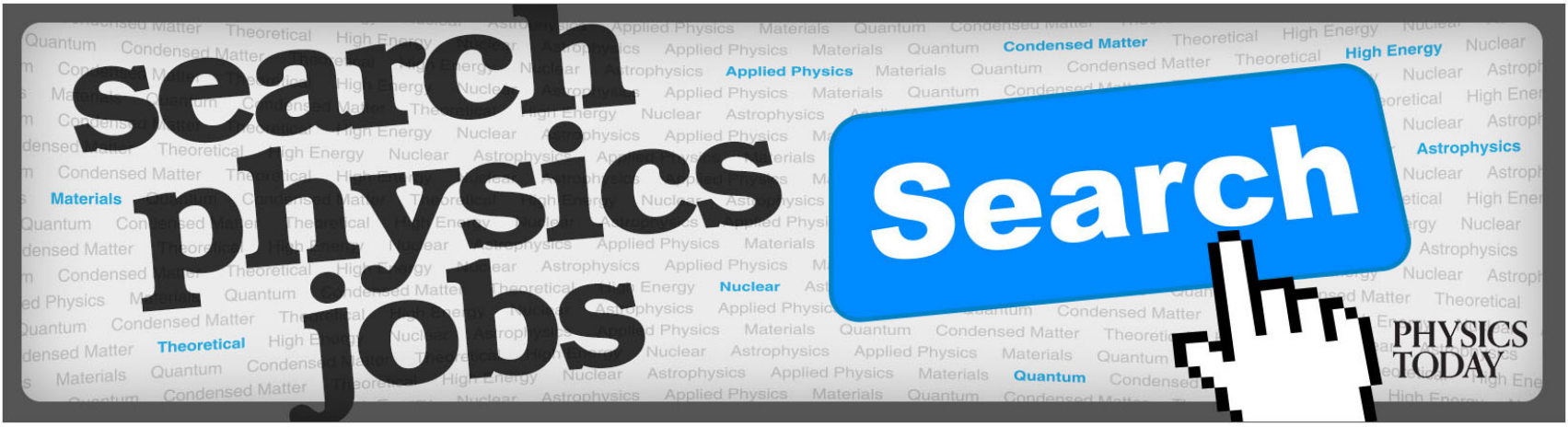




\title{
Non-diffusive resonant acceleration of electrons in the radiation belts
}

\author{
A. V. Artemyev, ${ }^{1, \text { a) }}$ V. V. Krasnoselskikh, ${ }^{1}$ O. V. Agapitov, ${ }^{1, b)}$ D. Mourenas,${ }^{2}$ and G. Rolland ${ }^{3}$ \\ ${ }^{1}$ LPC2E/CNRS, University of Orleans, Orleans, France \\ ${ }^{2} C E A, D A M, D I F, F-91297$ Arpajon, France \\ ${ }^{3}$ CNES, Toulouse, France
}

(Received 14 September 2012; accepted 16 November 2012; published online 6 December 2012)

We describe a mechanism of resonant electron acceleration by oblique high-amplitude whistler waves under conditions typical for the Earth radiation belts. We use statistics of spacecraft observations of whistlers in the Earth radiation belts to obtain the dependence of the angle $\theta$ between the wave-normal and the background magnetic field on magnetic latitude $\lambda$. According to this statistics, the angle $\theta$ already approaches the resonance cone at $\lambda \sim 15^{\circ}$ and remains close to it up to $\lambda \sim 30^{\circ}-40^{\circ}$ on the dayside. The parallel component of the electrostatic field of whistler waves often increases around $\lambda \sim 15^{\circ}$ up to one hundred of $\mathrm{mV} / \mathrm{m}$. We show that due to this increase of the electric field, the whistler waves can trap electrons into the potential well via wave particle resonant interaction corresponding to Landau resonance. Trapped electrons then move with the wave to higher latitudes where they escape from the resonance. Strong acceleration is favored by adiabatic invariance along the increasing magnetic field, which continuously transfers the parallel energy gained to perpendicular energy, allowing resonance to be reached and maintained. The concomitant increase of the wave phase velocity allows for even stronger relative acceleration at low energy $<50 \mathrm{keV}$. Each trapping-escape event of electrons of $\sim 10 \mathrm{keV}$ to $100 \mathrm{keV}$ results in an energy gain of up to $100 \mathrm{keV}$ in the inhomogeneous magnetic field of the Earth dipole. For electrons with initial energy below $100 \mathrm{keV}$, such rapid acceleration should hasten their drop into the loss-cone and their precipitation into the atmosphere. We discuss the role of the considered mechanism in the eventual formation of a trapped distribution of relativistic electrons for initial energies larger than $100 \mathrm{keV}$ and in microbursts precipitations of lower energy particles. (C) 2012 American Institute of Physics. [http://dx.doi.org/10.1063/1.4769726]

\section{INTRODUCTION}

Electron acceleration in the radiation belts due to the local mechanism of wave-particle resonant interaction has been actively studied during recent years [see reviews by Refs. 1-3]. Electron interaction with whistler waves is usually assumed to have a diffusive character and, thus, major investigations are carried out in the frame of the quasi-linear theory, see Refs. 4, 6, 7, and references therein. Moreover, most estimates of energy diffusion are based on the simplified approximation of the parallel (or quasi-parallel) propagation of whistler waves relative to the background magnetic field in the outer radiation belts.

There are several studies devoted to alternative approaches. First of all, we should mention the calculation of diffusion rates directly from the Hamiltonian equations of motion. ${ }^{8}$ Estimations of the effect of oblique whistler propagation on electron scattering and acceleration were obtained accordingly. ${ }^{2}$ The same approach was used to study cyclotron resonant acceleration of electrons by whistlers generated by a lightning discharge in the Earth's atmosphere. ${ }^{9}$ This mechanism assumes acceleration of particles moving in the direction opposite to the waves and takes into account the variation of the mean wave frequency inside the wave

\footnotetext{
${ }^{\text {a) }}$ Also at Space Research Institute, RAS, Moscow, Russia. Electronic mail: ante0226@gmail.com.

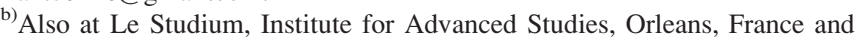
National Taras Shevchenko University of Kiev, Kiev, Ukraine.
}

packet. ${ }^{10}$ For whistler waves generated in the vicinity of the equatorial plane, such an interaction is possible for electrons moving from the mirror points toward the equator and it can result in a change of the direction of particle motion. ${ }^{11}$ It is also worth noting the self-consistent approach based on the calculation of individual particle trajectories, including the generation of whistler waves by an unstable electron population and the following interaction of these waves with resonant electrons. ${ }^{5}$ Still, most of the above-mentioned works consider quasi-parallel whistler waves.

Nonlinear mechanisms of particle acceleration in the radiation belts correspond to wave amplitudes exceeding a certain level determined by the inhomogeneity of the background magnetic field. ${ }^{12-14}$ Therefore, consideration of nonlinear wave-particle interaction requires existence of waves intense enough. Spacecraft observations of large-amplitude whistler waves have been recently reported. ${ }^{15-18}$ Although these observations are relatively rare, such waves can be responsible for very fast electron acceleration up to MeVs. ${ }^{11,19-21}$ The interaction of particles with largeamplitude waves propagating in the inhomogeneous magnetic field is indeed known to have a non-diffusive character including effects of capture (or phase trapping, see Refs. 2, 20, 22, and references therein).

Besides, the approximation of quasi-parallel whistler wave propagation relative to the background magnetic field is not supported by ray-tracing models at $L \sim 4-5 .^{23-25}$ Moreover, recent statistical analysis of spacecraft observations of 
wave-normal directions unambiguously demonstrated that whistler waves generated around the magnetic equator rapidly deviate from the local magnetic field direction. ${ }^{26-28}$ Already at $15^{\circ}-20^{\circ}$ of magnetic latitude $\lambda$, the angle $\theta$ between the whistler wavevector and the magnetic field can be larger $40^{\circ}$. Oblique propagation of whistler waves should result in a dominance of the electrostatic component. ${ }^{29,30}$ If the electrostatic component of large-amplitude whistler waves becomes strong enough at moderate magnetic latitude $\lambda \geq 15^{\circ}$ up to reflection points, then a substantial parallel electrostatic field could be present at these latitudes. The impact of such waves for non-diffusive electron acceleration is the subject of our study.

To develop a simple model of whistler wave describing evolution of the amplitude and wavevector in an inhomogeneous magnetic field, we rely on a statistical model based on spacecraft observations. We study the relativistic electron interaction with such a model wave and describe the nondiffusive process of rapid electron acceleration. Analytical estimates of electron trapping and acceleration are proposed and proved to be correct by comparisons with numerical calculations. We determine the efficiency of electron acceleration and estimate the effects of various mechanisms potentially capable of breaking the wave-particle resonant interaction.

\section{DESCRIPTION OF OBLIQUELY PROPAGATING WHISTLER WAVE}

High-amplitude whistler waves are generated in the vicinity of the magnetic equator and propagate along field lines, forming series of wave-packets, where each packet has a width of about $10^{2}-10^{3}$ periods. ${ }^{31} \mathrm{We}$ show the typical waveform of such wave-packets in Fig. 1. Here, upper limits of measurements are $40 \mathrm{mV} / \mathrm{m}$, but similar waveforms were observed with amplitude exceeding $100 \mathrm{mV} / \mathrm{m}$ (see, e.g., Refs. 16 and 18). One can see the series of wave-packets, each packet lasting about one second. Since this time is com-

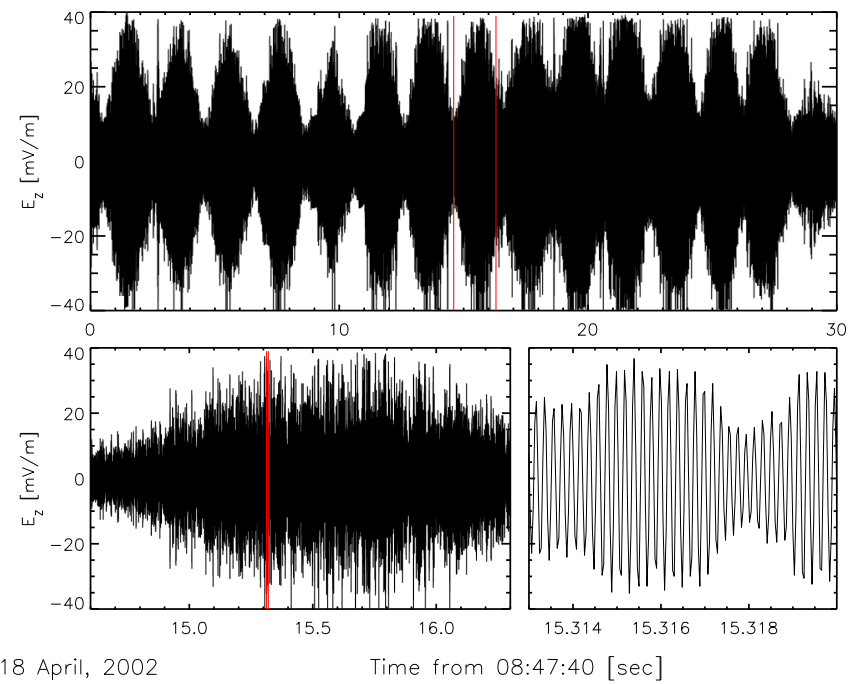

FIG. 1. Electric field waveform of chorus emissions registered aboard C4 Cluster spacecraft by wide-band data (WBD) plasma wave instrument. ${ }^{32}$ The amplitude limit of measurements is $40 \mathrm{mV} / \mathrm{m}$. The angle $\theta$ of wavenormal direction estimated from the Spatio-Temporal Analysis of Field Fluctuations-Spectrum Analyzer (STAFF-SA) experiment ${ }^{33}$ is about $30^{\circ}$. parable with the period of electron bounce oscillations, a single wave-packet fills the whole field line along electron trajectory.

According to recent spacecraft observations, the mean value of the $\theta$ wave-normal angle distribution increases with magnetic latitude, ${ }^{27}$ at least on the dayside at $L$-shells from 4 to 5 . We use a simplified model of $\theta$ variation with latitude, based on Cluster statistics, where the dependence $\theta(\lambda)$ can be approximated by the function

$$
\theta(\lambda) \approx\left\{\begin{array}{l}
\left(\theta_{r}\left(15^{\circ}\right)-\theta_{\Delta}\right) \cdot\left(\lambda / 15^{\circ}\right) \\
\left(\theta_{r}(\lambda)-\theta_{\Delta}\right) \cdot\left(3 / 2-\lambda / 30^{\circ}\right),
\end{array}\right.
$$

where $\theta_{\Delta}=10^{\circ}$ and $\theta_{r}(\lambda)$ is angle of the resonant cone, i.e., $\cos \theta_{r}(\lambda)=\omega / \Omega_{c}(\lambda)$ with $\omega$ the wave frequency and $\Omega_{c}$ $=\Omega_{c}(\lambda)$ the electron gyrofrequency. Here, we use a dipolar magnetic field model $B_{0}(\lambda)=B_{e q} \sqrt{1+3 \sin ^{2} \lambda} / \cos ^{6} \lambda$ with equatorial value $B_{e q}$ and corresponding equatorial gyrofrequency $\Omega_{c 0}$. The function $\theta(\lambda)$ is shown in Fig. 2 (left panel) for two typical values of ratio $\omega / \Omega_{c 0}=0.2$ and $\omega / \Omega_{c 0}$ $=0.35$.

Whistler waves propagate along field lines until reflection latitude $\lambda^{*}$, where their frequency reaches local lowerhybrid frequency $\Omega_{L H} \approx \Omega_{c} \sqrt{m_{e} / m_{i}}$ (here, $m_{e} / m_{i}$ is ratio of electron mass and effective mass of ion mixture). Far from the reflection point, the dispersion relation of whistler waves can be written as ${ }^{29}$

$$
\omega=\Omega_{c} \cos \theta \frac{k^{2} c^{2}}{k^{2} c^{2}+\Omega_{p e}^{2}},
$$

where plasma frequency $\Omega_{p e}$ is assumed to be larger than gyrofrequency and constant along field lines (the approximation $\Omega_{p e}=$ const is valid for $\lambda<35^{\circ}$, see Ref. 34). Using dispersion relation and reflection condition $\omega \sim \Omega_{L H}$, we obtain estimates for reflection latitude $\cos \theta\left(\lambda^{*}\right) \approx \sqrt{m_{e} / m_{i}}$. For proton dominated plasma, we have $\lambda^{*} \approx 41^{\circ}$ and $\lambda^{*} \approx 46^{\circ}$ for $\omega / \Omega_{c 0}=0.2$ and $\omega / \Omega_{c 0}=0.35$, respectively.

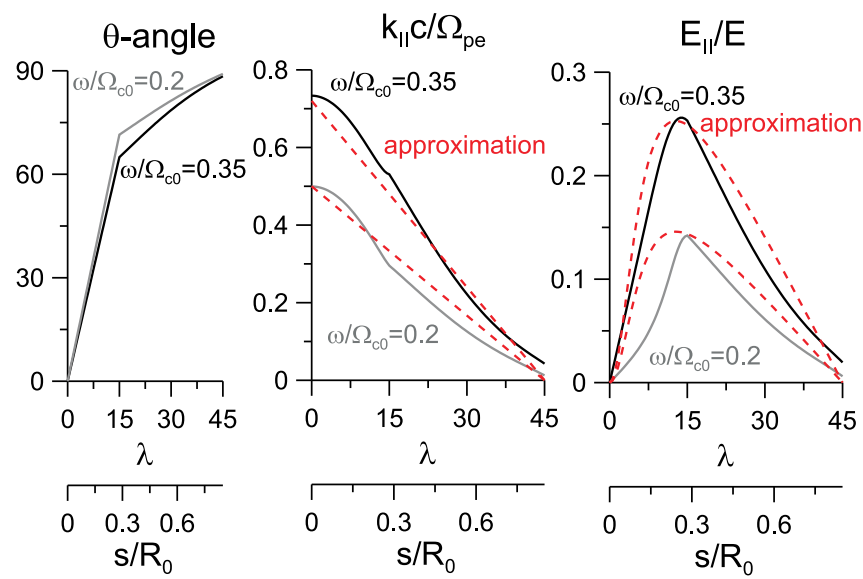

FIG. 2. Approximation of $\theta$ as function of magnetic latitude is shown in left panel. Central panel shows the parallel component of wavevector $k_{\|}$and the corresponding approximation (red color). Right panel shows the normalized parallel component of electric field $E_{\|} / E$ and the corresponding approximation (red color) 
The combination of the dispersion relation and the expression for $\theta(\lambda)$ gives the dependence of the parallel component of wavevector $k_{\|}$on $\lambda$ (see Fig. 2, central panel). $k_{\|}$decreases monotonously with $\lambda$. The ratio of the parallel component of the electric field and its total amplitude $E_{\|} / E$ strongly varies with $\theta$, see Ref. 29. As a result, we can plot $E_{\|} / E$ as function of $\lambda$ by taking into account expression for $\theta=\theta(\lambda)$ and dispersion relation (see Fig. 2, right panel). Below $\lambda=15^{\circ}, E_{\|}$is growing due to the increase of $\theta$. This growth can be enhanced by a general increase of electric field amplitude with $\lambda$ (see, e.g., Refs. 6 and 35). Above $\lambda=15^{\circ}$, wave-normal angle $\theta$ approaches the resonance cone $\theta_{r}$ value and the whistler mode becomes electrostatic with parallel component of electric field $E_{\|} \approx E \cos \theta \sim \cos \theta$. Thus, the parallel component has a local maximum around $\lambda=15^{\circ}$ and then decreases (see Fig. 2, right panel).

For both functions of wavenumber and electrostatic field, we use in Fig. 2 a second dependence upon $s$ - arclength along the field line: $d s / d \lambda=R_{0} \sqrt{1+3 \sin ^{2} \lambda} \cos \lambda$, where $R_{0} \approx R_{E} L$ is the curvature radius of field lines, $R_{E} \approx$ $6400 \mathrm{~km}$ is the Earth radius, and $L$ is shell number. Dependencies of $k_{\|}$and $E_{\|}$on $s$ can be approximated as

$$
\begin{aligned}
k_{\|} & \sim 1-\left|s / s^{*}\right| \\
E_{\|} & \sim \eta(s)\left(1-\left|s / s^{*}\right|\right),
\end{aligned}
$$

where $s^{*} \approx 0.85 R_{0}$ corresponds to the reflection point $\lambda^{*}$ and function $\eta(s)=s^{2} /\left(s^{2}+\right.$ const $)$. These approximations are shown in Fig. 2 as well. We also introduce $s_{0}=0.25 R_{0}$ corresponding to $\lambda=15^{\circ}$, where $E_{\|}$has local maximum (it can be mentioned, that $\eta \approx 1$ for $s>s_{0}$ ).

\section{EQUATIONS OF PARTICLE MOTION}

We describe the wave-particle resonant interaction for a relativistic electron with mass $m_{e}$ and charge $-e$. We assume that the magnetic field changes slowly and that the first adiabatic invariant is conserved. While cyclotron resonance interaction with high-amplitude waves usually leads to the destruction of the first adiabatic invariant, ${ }^{37,42}$ it is worth emphasizing that we consider here Landau resonance interaction which preserves the first adiabatic invariant (e.g., see the review of wave-particle interactions in Ref. 2). Thus, we can average the Hamiltonian over gyrorotation and obtain ${ }^{36}$

$$
H=\sqrt{m_{e}^{2} c^{4}+c^{2} p_{\|}^{2}+c^{2} \mu B_{0}(s)}-e \Phi(s, t),
$$

where $\left(s, p_{\|}\right)$are parallel components of coordinate and particle momentum, $B_{0}(s)$ is the magnetic field with equatorial value $B_{e q}=B_{0}(0), \Phi(s, t)$ is the scalar potential of the wave (i.e., $\partial \Phi(s, t) / \partial s=-E_{\|}$, with wave electric field $\left.E_{\|}\right)$. Electron magnetic moment can be written as $\mu=m_{e} c^{2}\left(\gamma_{0}^{2}-1\right)$ $\sin ^{2} \alpha_{0} / B_{e q}$, where $\gamma_{0}$ is the initial value of relativistic factor $\gamma=(H+e \Phi(s, t)) / m_{e} c^{2}$, and $\alpha_{0}$ is the equatorial value of pitch-angle. Hamiltonian equations of electron motion take the form

$$
\begin{aligned}
m_{e} \gamma \dot{s} & =p_{\|} \\
\gamma \dot{p}_{\|} & =-\frac{\mu}{2 m_{e}} \frac{d B_{0}(s)}{d s}-e E_{\|}(s, t) \gamma \\
\phi & =\int_{0}^{s} k_{\|}(\tilde{s}) d \tilde{s}-\omega t+\phi_{0} .
\end{aligned}
$$

Using the approximation of parallel wave-number $k_{\|}$, we can rewrite the first term of the wave phase

$$
\begin{aligned}
& \int_{0}^{s} k_{\|}(\tilde{s}) d \tilde{s}=k_{0} \int_{0}^{s}\left(1-\left|\tilde{s} / s^{*}\right|\right) d \tilde{s}=K(s) \\
& K(s)=\kappa\left(s / s^{*}\right)\left(1-\frac{1}{2}\left|s / s^{*}\right|\right),
\end{aligned}
$$

where $\kappa=k_{0} s^{*}$ and $k_{0}=$ const is the equatorial amplitude of wavenumber. For the following numerical calculations, we define $k_{0}$ using equatorial value of refractive index $(k c / \omega)_{s=0}$, where typical frequency of whistler-waves in radiation belts is $\omega=0.35 \Omega_{c 0}$ and for $L$-shell around 5 we have $\omega \approx 3 \mathrm{kHz}$. We use a simplified approximation for the magnetic field variations that is valid in the vicinity of the equator: $B_{0}(s)=1+(9 / 2)\left(s / R_{0}\right)^{2}$. Now, we rewrite the system of equations (1) in dimensionless form

$$
\left\{\begin{array}{l}
\gamma \dot{s}=p_{\|} \\
\gamma \dot{p}_{\|}=-\frac{9}{2} \Omega_{b}^{2} s-\gamma \varepsilon(1-|s|) \eta(s) G(\phi) \\
\phi=K(s)-\omega t+\text { const } \\
K(s)=\kappa s\left(1-\frac{1}{2}|s|\right)
\end{array}\right.
$$

where $\quad \gamma=\sqrt{1+p_{\|}^{2}+\Omega_{b}^{2}\left(1+\frac{9}{2} s^{2}\right)}, \Omega_{b}=\sqrt{\gamma_{0}^{2}-1} \sin \alpha_{0}$, $s / R_{0} \rightarrow s, p_{\|} / m_{e} c \rightarrow p_{\|}, t c / R_{0} \rightarrow t, \omega R_{0} / c \rightarrow \omega, \quad \varepsilon=$ $e \tilde{E}_{0} R_{0} / m_{e} c^{2}, \tilde{E}_{0}=0.45 E_{0}$ and $E_{0}$ is amplitude of wave electric field (factor 0.45 corresponds to the maximum value of ratio $E_{\|} / E \approx 0.26$ taken from Fig. 2 for system with $\omega / \Omega_{c 0}=0.35$, i.e., $\left.0.45 \cdot \max (\eta(s)(1-|s|)) \approx 0.26\right)$. We also assume that $s^{*} \approx R_{0}$. Function $G(\phi)$ defines the phasedependence of the wave. Here, we consider periodical wave packets: each packet consists of one hundred periods and the distance between two successive packets is equal to two hundred periods of wave, i.e., $G(\phi)=\exp \left(-5 \cos ^{2}(\sigma \phi)\right) \cos \phi$ with $\sigma=0.01$ (see scheme in Fig. 3).

\section{RESONANT WAVE-PARTICLE INTERACTION: ANALYTICAL ESTIMATES}

We consider the effect of electron trapping using an analytical approach. Particle can be trapped in the vicinity of the Landau resonance $\dot{\phi}=0$, where one can write

$$
\dot{\phi}=\kappa(1-s)\left(p_{\|} / \gamma\right)-\omega .
$$

Here, for simplicity, we consider the half-space $s>0$. We introduce resonant velocity $v_{R}=(\omega / \kappa)(1-s)^{-1}$ and resonant 


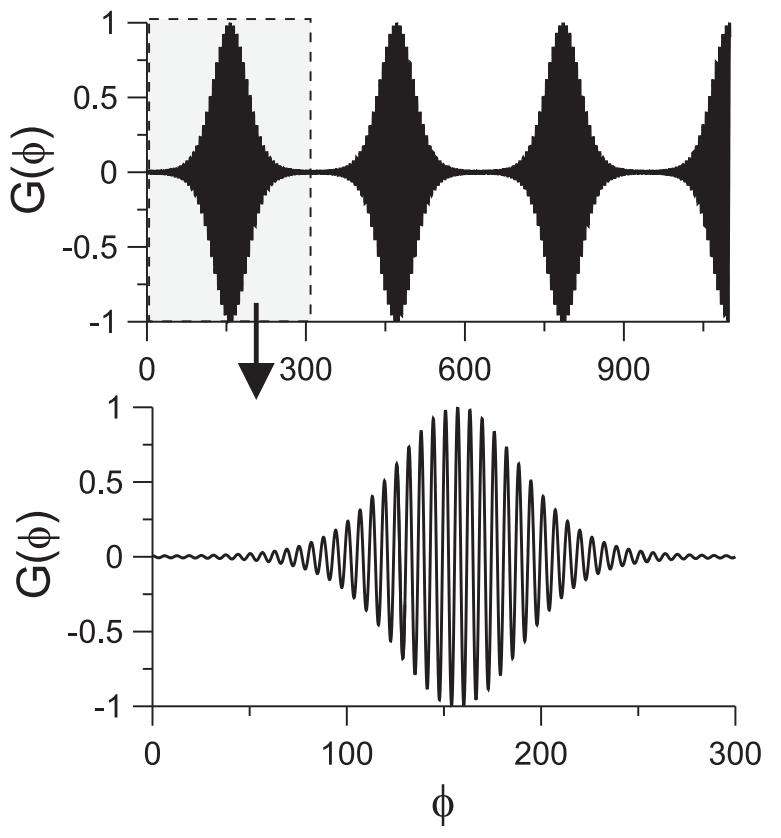

FIG. 3. Schematical view $G(\phi)$ function.

relativistic factor $\gamma_{R}=1 / \sqrt{1-v_{R}^{2}}$ and rewrite system (2) as follows

$$
\begin{aligned}
\gamma_{R}^{2} \gamma^{2}(\ddot{\phi} / \kappa)= & -\gamma_{R}^{2}(1-s)\left(\gamma^{2} \dot{v}_{R}+\frac{9}{2} \Omega_{b}^{2} s\right) \\
& -\gamma \varepsilon(1-s)^{2} \eta(s) \cos \phi .
\end{aligned}
$$

For analytical estimates, we assume that the electron interacts with the wave-packet close to its central region, where $G(\phi) \sim \cos \phi$. Resonant velocity of the wave is always much smaller than the speed of light and we can take $\gamma_{R} \approx 1$. In this case, the relativistic factor for resonant electron can be written as

$$
\gamma \approx \sqrt{1+\Omega_{b}^{2}\left(1+(9 / 2) s^{2}\right)}
$$

Finally, we obtain an equation for the wave phase $\phi$ in the resonance

$$
\left\{\begin{array}{l}
\gamma^{2} \ddot{\phi} / \kappa=-\mathrm{A}(s)-\mathrm{B}(s) \cos \phi \\
\mathrm{A}(s)=\frac{\omega^{2}}{\kappa^{2}} \gamma^{2}(1-s)^{-2}+\frac{9}{2} \Omega_{b}^{2} s(1-s) \\
\mathrm{B}(s)=\gamma \varepsilon(1-s)^{2} \eta(s) .
\end{array}\right.
$$

System (3) is similar to equations derived in Refs. 22, 37, and 38 (see also original papers Refs. 12, 13, 39, and review Ref. 2). Here, $\kappa$ and $\omega$ are supposed to be large (i.e., wave-frequency is substantially higher than the frequency of electron bounce oscillations), thus one can consider variables $(\phi, \gamma \dot{\phi})$ as fast ones and variable $s$ as a slow one. The equation of motion can then be written as $\ddot{\phi}+d U / d \phi=0$, where $U(\phi)$ plays the role of potential energy. In this case, Eq. (3) corresponds to Hamiltonian

$$
H_{\phi}=\frac{1}{2} \gamma^{2} \dot{\phi}^{2}+\kappa(\mathrm{A}(s) \phi+\mathrm{B}(s) \sin \phi) .
$$
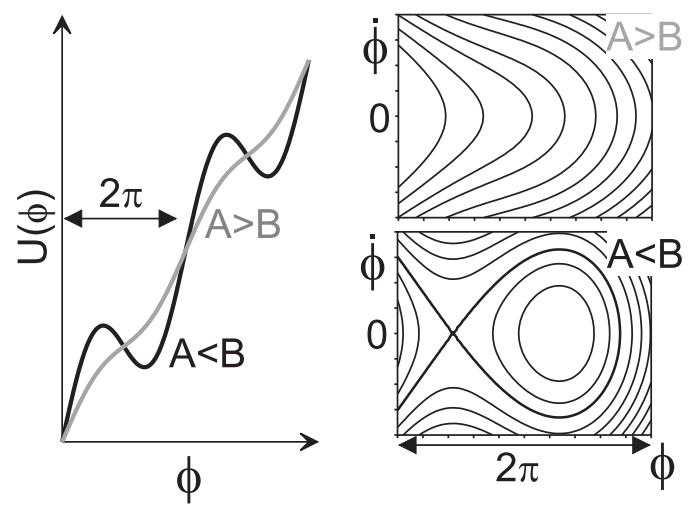

FIG. 4. Panels show profiles of potential energy $U(\phi)$ and phase portraits for systems with $\mathrm{A}<\mathrm{B}$ and with $\mathrm{B}<\mathrm{A}$. In the phase portrait with $\mathrm{A}<\mathrm{B}$, the separatrix is shown by black solid curve.

In Fig. 4, we plot profiles of potential energy $U(\phi)=H_{\phi}$ $-(1 / 2) \gamma^{2} \dot{\phi}^{2}$ and phase portraits of the system for $\mathrm{A}>\mathrm{B}$ and $\mathrm{A}<\mathrm{B}$. In the system with $\mathrm{A}<\mathrm{B}$, one can find local potential minima and related regions with closed trajectories in the $(\phi, \gamma \dot{\phi})$ plane. Particles on these trajectories move with the wave, because their $\dot{\phi}$ oscillates around zero value. Here, $(\kappa \mathrm{B})^{1 / 2}$ is equivalent to frequency of oscillations of trapped particle and $\kappa \mathrm{A}$ depends on the system inhomogeneity along field lines. For constant $\mathrm{B}$ and $\mathrm{A}$, Hamiltonian $H_{\phi}$ is a constant of motion.

There is a separatrix in the phase plane $(\phi, \gamma \dot{\phi})$ for $\mathrm{A}<\mathrm{B}$ - the curve separating the region with closed trajectories from the other phase space. A particle can become trapped inside the region with closed trajectories only if the area surrounded by the separatrix $S$ grows with time. ${ }^{40}$ The corresponding physical process consists in trapping of particles by the wave due to increase of its amplitude ${ }^{41}$ or due to decrease of effect of the magnetic field inhomogeneity. ${ }^{37,42}$ In Appendix A, we show that wave can trap particles in the region $|s|<s_{0}$, while particles are released from the trapping regime in $|s|>s_{0}$.

Trapping of electrons into resonance is possible if $\mathrm{A}<\mathrm{B}$ and Landau resonance condition $p_{\|} / \gamma \approx v_{R}>0$ is satisfied. Escape from the resonance corresponds to the moment, when $\mathrm{A} \approx \mathrm{B}$ and $d \mathrm{~A} / d s>d \mathrm{~B} / d s$ for particle with $\dot{s}>0$ (actually, conditions of particle escape from the resonance are more complicated and are determined by the value of the area surrounded by the trajectory in the plane $(\phi, \gamma \dot{\phi})$ just before the trapping [see Ref. 40], but one can use the simplified condition $\mathrm{A} \approx \mathrm{B}$ as a first order approximation). Profiles of coefficients $\mathrm{A}$ and $\mathrm{B}$ as functions of $s$ are shown in Fig. 5. Regions where resonant condition $p_{\|} / \gamma \approx v_{R}$ is satisfied are filled by colors. Trapped electrons should leave the resonance at the points marked by arrows in Fig. 5, where $\mathrm{A}=\mathrm{B}$. Therefore, resonant trapping and acceleration is possible only for particles with equatorial pitch-angles inside a certain range $\alpha_{0} \in\left[\alpha_{\min }, \alpha_{\max }\right]$.

The minimum value $\alpha_{\text {min }}$ is defined by the resonant condition $p_{\|} / \gamma \approx v_{R}$, i.e., with the decrease of $\alpha_{0}$, the position of the resonance goes to higher latitude and eventually occurs in the region where $d \mathrm{~B} / d s<0$ (electrons with $\alpha_{0} \approx \alpha_{\min }$ are shown in Fig. 5 by red color). Increase of energy shifts the resonant condition to higher latitudes as well, e.g., for 

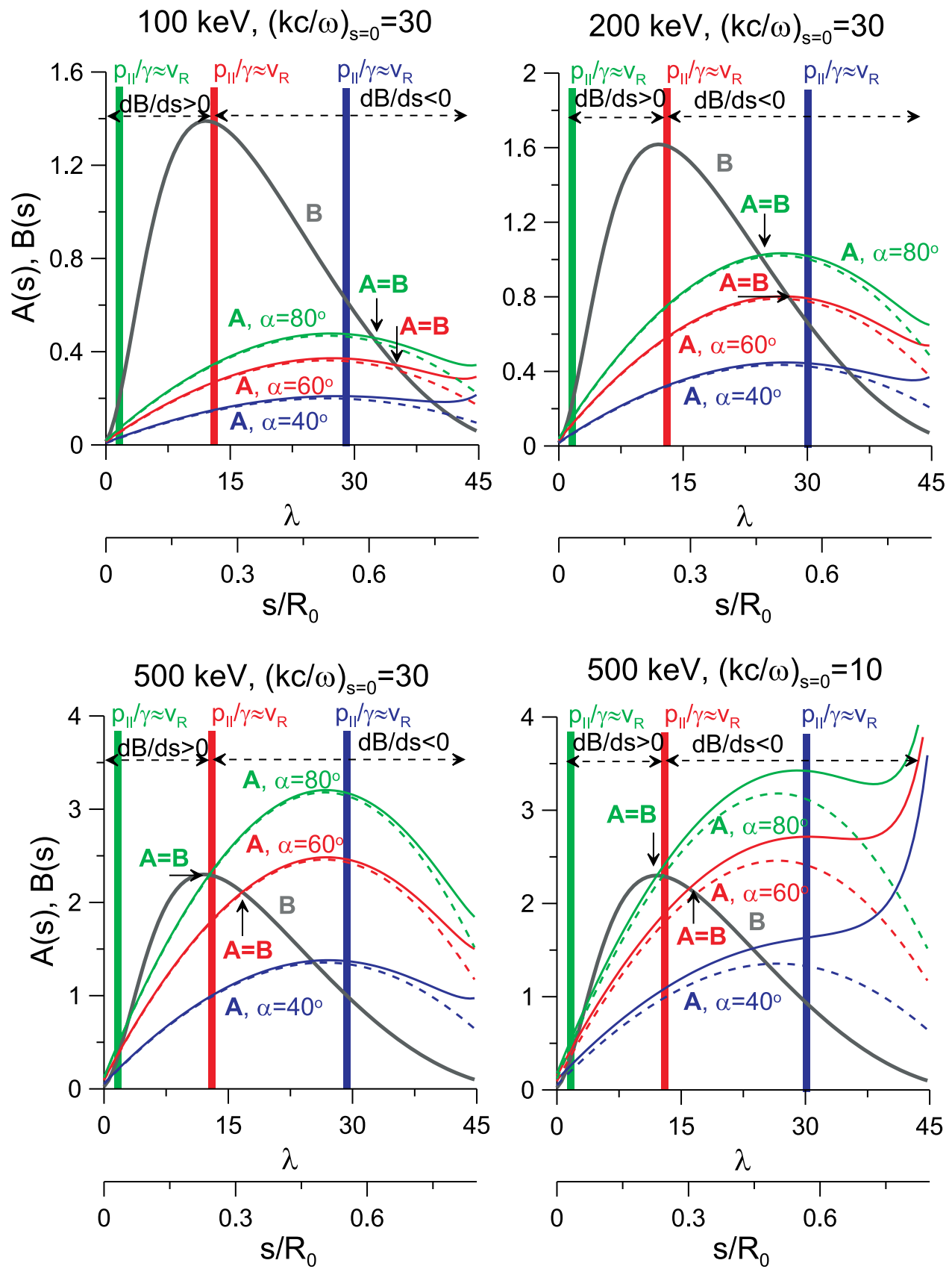

FIG. 5. Coefficients A, B as function of $s$ and $\lambda$ for various particle energy and pitch-angle $\alpha_{0}$ (dashed curve corresponds to A calculated without $(\gamma \omega / \kappa)^{2}$ $(1-s)^{-2}$ term). Colored regions correspond to the resonant condition $p_{\|} / \gamma$ $\approx v_{R}$, see text for details. All panels correspond to $E_{0}=100 \mathrm{mV} / \mathrm{m}$. Regions of escape from the resonance with $s=s_{\text {esc }}$ (where, $\mathrm{A}=\mathrm{B}$ and $d \mathrm{~B} / d s<0$ ) are shown by solid arrows. Dashed arrows indicate regions with $d \mathrm{~B} / d s>0$ and $d \mathrm{~B} / d s<0$.
$100 \mathrm{keV}$ we have $\alpha_{\min } \approx 60^{\circ}$ and for $500 \mathrm{keV}, \alpha_{\min } \approx 70^{\circ}$. One example of resonant condition located in the region with $d \mathrm{~B} / d s<0$ is shown by blue color in Fig. 5 .

The maximum value $\alpha_{\max }$ corresponds to the lower boundary of the region where $\mathrm{B}>\mathrm{A}$. For particles with energy less than $1 \mathrm{MeV}$ and reasonable values of equatorial refractive index $(k c / \omega)_{s=0}$, we have $\alpha_{\max } \approx 80^{\circ}$ (shown in Fig. 5 by green color). Electrons with $\alpha_{0}>\alpha_{\max }$ have resonant condition $p_{\|} / \gamma \approx v_{R}$ satisfied in the vicinity of the equator, where $\mathrm{B}<\mathrm{A}$ and trapping is impossible.

Fig. 5 shows that particles can be trapped in the vicinity of the equator and then should escape from the resonance around a point $s \approx s_{\text {esc }}$. All the energy gained corresponds to the difference between the initial coordinate of trapping and $s_{\text {esc }}: \Delta \gamma \approx\left(\partial \gamma / \partial v_{R}\right) \Delta v_{R}+\left(\partial \gamma / \partial B_{0}\right) \Delta B_{0}$. The first term is provided by the variation of wave phase velocity $v_{R}(s)$ and the second term corresponds to magnetic field inhomogeneity.
Both these effects determine $s_{\text {esc }}$ and also the input into $\Delta \gamma$. However, here we suppose that $\gamma_{R} \approx 1$ and, as a result, $\partial \gamma / \partial v_{R}=0$. In this case, all the energy gain is provided by trapped particle acceleration along an increasing magnetic field. As an electron travels along a field line, the conservation of the magnetic moment leads to a continual transfer of parallel energy to perpendicular energy. After trapping, it allows for resonant acceleration over a large distance by continuously storing away part of the parallel energy gained by the particle, under the form of perpendicular energy. The related inhomogeneity of the phase velocity $v_{R}(s)$ can also influence the particle acceleration by changing the coordinate of escape $s_{\text {esc }}$, which is defined by equation $\mathrm{A}\left(s_{\text {esc }}\right)=\mathrm{B}\left(s_{\text {esc }}\right)$. For high energy particles $(>100 \mathrm{keV})$, we can neglect the first term corresponding to inhomogeneity of $v_{R}(s)$ in $\mathrm{A}(s)$ (one can see that $\mathrm{A}(s)-(\gamma \omega / \kappa)^{2}(1-s)^{-2}$ shown in Fig. 5 by dashed curves is very close to full $\mathrm{A}(s)$ in the vicinity of $\left.s_{e s c}\right)$. At high energies, 
indeed, the effect of $v_{R}(s)$ variation with $s$ appears negligible. In this case, we obtain the following expression for $s_{\text {esc }}$ from equation $\mathrm{A}\left(s_{\text {esc }}\right)=\mathrm{B}\left(s_{\text {esc }}\right)$

$$
s_{\text {esc }}=\left(\frac{9}{2} \frac{\Omega_{b}^{2}}{\gamma \varepsilon}+1\right)^{-1},
$$

where we use the approximation $\eta\left(s_{\text {esc }}\right) \approx 1$, because $s_{\text {esc }}>s_{0}$. The corresponding energy gain is

$$
\Delta \gamma \approx \frac{9}{4} \Omega_{b}^{2} s_{e s c}^{2} / \gamma \approx \frac{9}{4} \Omega_{b}^{2} \gamma^{-1}\left(\frac{9}{2} \frac{\Omega_{b}^{2}}{\gamma \varepsilon}+1\right)^{-2} .
$$

If we consider the first trapping of a particle, then we should take into account the dependence of $\Omega_{b}$ on $\gamma_{0}$ and rewrite the expression for $\Delta \gamma$

$$
\Delta \gamma \approx \frac{9}{4} \sin ^{2} \alpha_{0} \frac{\gamma_{0}^{2}-1}{\gamma_{0}}\left(\frac{9}{2} \frac{\sin ^{2} \alpha_{0}}{\varepsilon} \frac{\gamma_{0}^{2}-1}{\gamma_{0}}+1\right)^{-2} .
$$

The maximum value of $\Delta \gamma_{\max }$ corresponds in this case to $\left(\gamma_{0}^{2}-1\right) / \gamma_{0}=\frac{2}{9} \sin ^{-2} \alpha_{0} \varepsilon$ and $\Delta \gamma_{\max }=\varepsilon / 8 \quad$ (e.g., for $E_{0}=100 \mathrm{mV} / \mathrm{m}$, we have a maximum energy gain around $\sim 150 \mathrm{keV})$.

At small initial energies $E \sim 10 \mathrm{keV}$, the inhomogeneity of the phase velocity $v_{R}(s)$ (related to the magnetic field inhomogeneity) must be considered, as it modifies sensibly the location of escape $s_{\text {esc }}$. In this case, one obtains $\Delta \gamma \sim \varepsilon^{3 / 4}(\omega / \kappa)^{1 / 2}$, corresponding to an even higher relative energy gain (e.g., for $E_{0}=100 \mathrm{mV} / \mathrm{m}$, the energy gain can attain $\sim 50 \mathrm{keV}$, see Appendix C).

\section{RESONANT WAVE-PARTICLE INTERACTION: NUMERICAL CALCULATIONS}

We compare our estimates with the full numerical solution of system (2) for particles with various initial energies and pitch-angels. We take wave-amplitude $E_{0}=100 \mathrm{mV} / \mathrm{m}$ and $(k c / \omega)=30$ at the equator, while $L$-shell is equal to 5 . Unit of modelling time $R_{0} / c$ is about $0.1 \mathrm{~s}$. Evolution of particle energy and a fragment of particle trajectory are shown in Fig. 6. Initially, the particle moves along a closed trajectory in $\left(s, p_{\|}\right)$plane. This periodical motion corresponds to bounce oscillations. Then, the particle becomes trapped by the wave and gains energy. This gain corresponds to its motion from one initially closed trajectory to another closed trajectory with larger radius. Energy gain for one capture is about $100 \mathrm{keV}$, in agreement with analytical estimates. Also, small variations of particle energy can be observed before and after the energy jump due to scattering effect.

When a given particle passes through the resonance (i.e., $p_{\|} / \gamma=v_{R}$ ), it can be trapped (if $\mathrm{A}<\mathrm{B}$ and $d \mathrm{~B} / d s$ $>0$ ). However, trapping is a probabilistic process with probability $\mathrm{P} \ll 1$, see Appendix A. If a particle is not trapped it is scattered: small quasi-random jumps of energy occur. The mean value of these jumps is equal to zero, but jumps provide energy diffusion. ${ }^{22,37,42}$ Bottom panel of Fig. 6 shows a fragment of particle trajectory in the plane $(\phi, \gamma \dot{\phi})$. This fragment corresponds to particle motion after trapping,
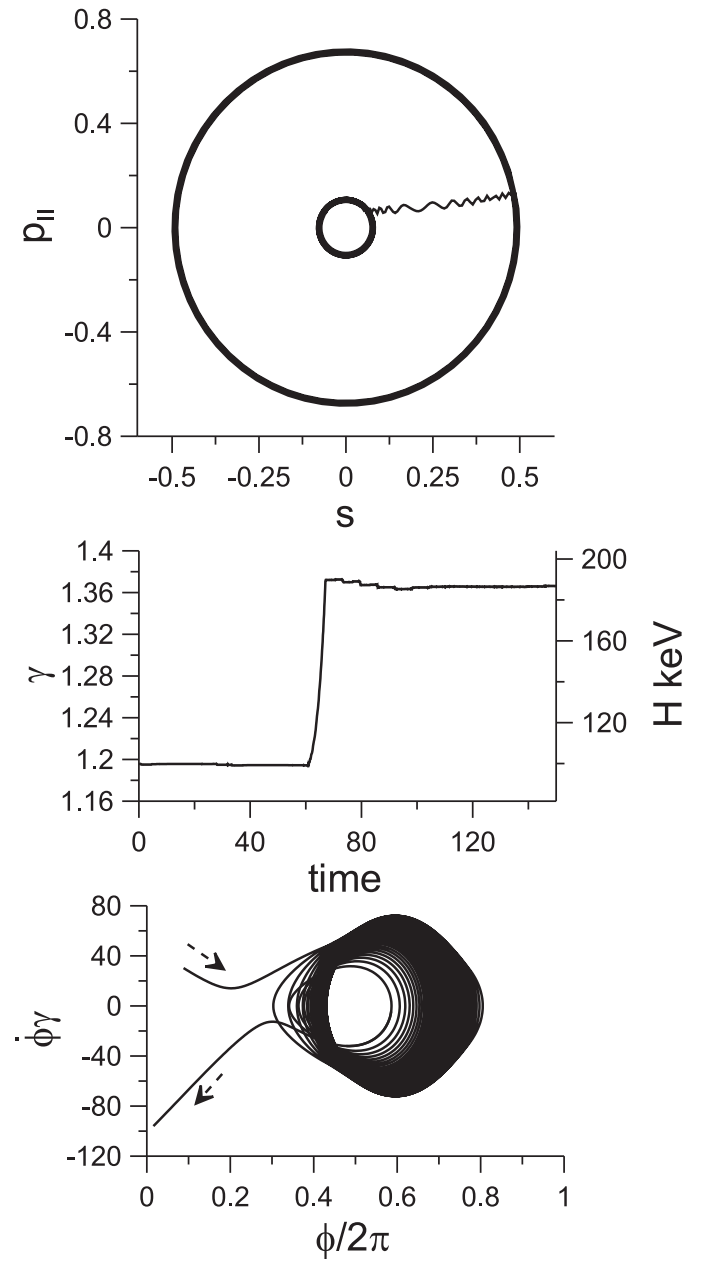

FIG. 6. Top panel displays particle trajectory in $\left(s, p_{\|}\right)$plane and middle panel shows particle energy (and relativistic factor) as function of time. Bottom panel shows fragment of particle trajectory in $(\phi, \gamma \dot{\phi})$ plane. Initial value of equatorial pitch-angle is $\alpha_{0}=80^{\circ}$.

when $\dot{\phi}$ oscillating around zero value. One can notice the similarity of the particle trajectory and the phase portrait in Fig. 4.

To illustrate the effects of varying values of wavenumbers $k_{0}$, initial energy, pitch-angle, and wave amplitude $E_{0}$, we plot energy as a function of time for several test-cases in Fig. 7. One can see that particle with $\alpha_{0}=80^{\circ}$ gains more energy for the same system parameters as compared with particle with $\alpha_{0}=60^{\circ}$. This effect corresponds to the different distances over which condition of trapping $(\mathrm{B}>\mathrm{A})$ is satisfied for various $\alpha_{0}$, see Fig. 5. Increase of wave amplitude $E_{0}$ leads to the increase of energy gained during one trapping-release event, while decrease of $k_{0}$ corresponds to the increase of probability of trapping (see Appendix A): for the same time interval, a particle can be trapped more times for smaller $k_{0}$ (smaller equatorial value of $k c / \omega$ ). If the initial energy is $500 \mathrm{keV}$, then each trapping-release event results in a relatively small energy gain. However, due to several successive trappings, a particle can increase its energy from $500 \mathrm{keV}$ up to $700 \mathrm{keV}$ during a time period of about a few minutes. We indicate several jumps of energy for a particle with initial $E=500 \mathrm{keV}$ and show the corresponding fragments of trajectory in $(\phi, \dot{\phi} \gamma)$ plane in Fig. 8. 

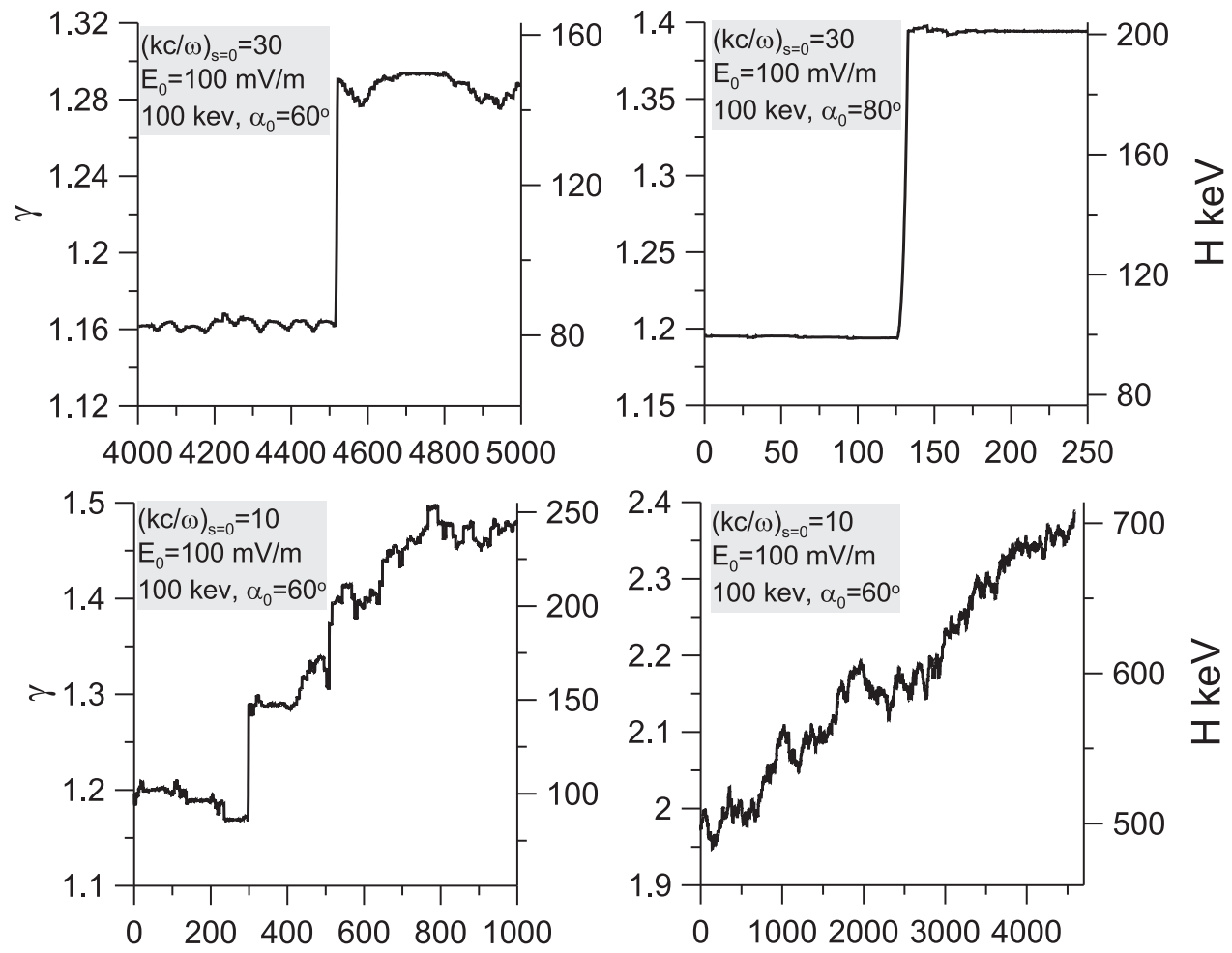

FIG. 7. Jumps of particle energy for various system parameters.
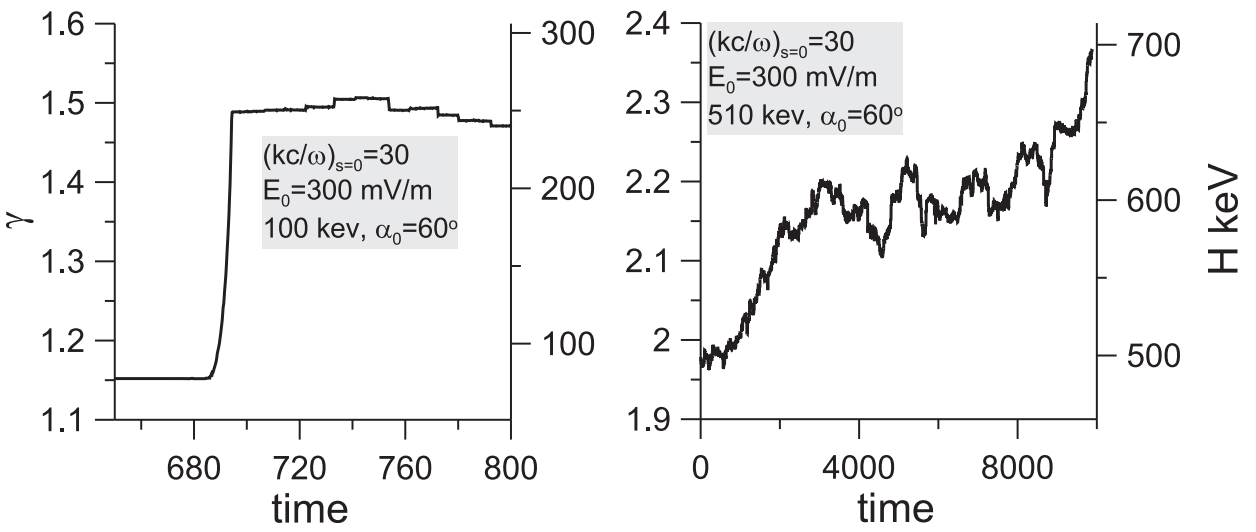

Each energy jump can be directly compared with trapped motion. However, in contrast with Fig. 6, where particle motion in the $(\phi, \dot{\phi} \gamma)$ plane corresponds to numerous rotations, here we have only a single rotation (half-rotation) in the course of trapped motion for Fig. 8 .

\section{EFFECTS LIMITING EFFICIENCY OF ACCELERATION}

Let us consider different effects which might limit the efficiency of the acceleration process. Whistler waves with $\theta$ close to $\theta_{r}$ propagate with a large angle relative to the magnetic field lines. As a result, such waves should leave the initial field line after a certain time, which can be estimated as the ratio of the transverse scale of wave-packet and the whistler velocity $\sim(\omega / k) \sin \theta_{r} \sim(\omega / k) \sim 10^{3}-10^{4} \mathrm{~km} / \mathrm{s}$. Transverse scale of wave-packet is about $500 \mathrm{~km}$ (see Ref. 43, and also reference therein) and we obtain an upper bound for the available time of wave-particle interaction as $\sim 0.1 \mathrm{~s}$. This time is similar to (or a little bit smaller than) the bounce period of particle oscillations and therefore particles have enough time to get trapped and escape with energy gain. However, the corresponding limitation of the available time for resonant interaction could result in a reduction of energy gain by a factor two-three.

Another effect corresponds to the actual width of wavepackets: according to Polar spacecraft data, ${ }^{31}$ coherent wavepackets are observed near the equator for about 100 periods duration (as we use in our calculations), but above $25^{\circ}$ latitude only several periods of coherency are usually found. Trapped particles move with a certain wave inside the wavepacket and if this wave conserves its properties till high latitudes, our estimates remain correct. However, if wave with trapped particles disappears due to dispersion or superposition effects, then particles should escape from the resonance before $|s| \sim s_{\text {esc }}$. To estimate this effect, we use a model taking into account the variation of the wave-packet width $G(\phi, s)=\exp \left(-5 \cos ^{2}(\sigma(s) \phi)\right) \cos \phi$, where $\sigma(s)=$ $0.01(1+9|s|)$ (corresponding wave-packets in the equatorial plane and at $|s|=1$ are shown in Fig. 9). Such variation of the wave-packet width reduces the number of coherent waves from one hundred to a single wave. This really 

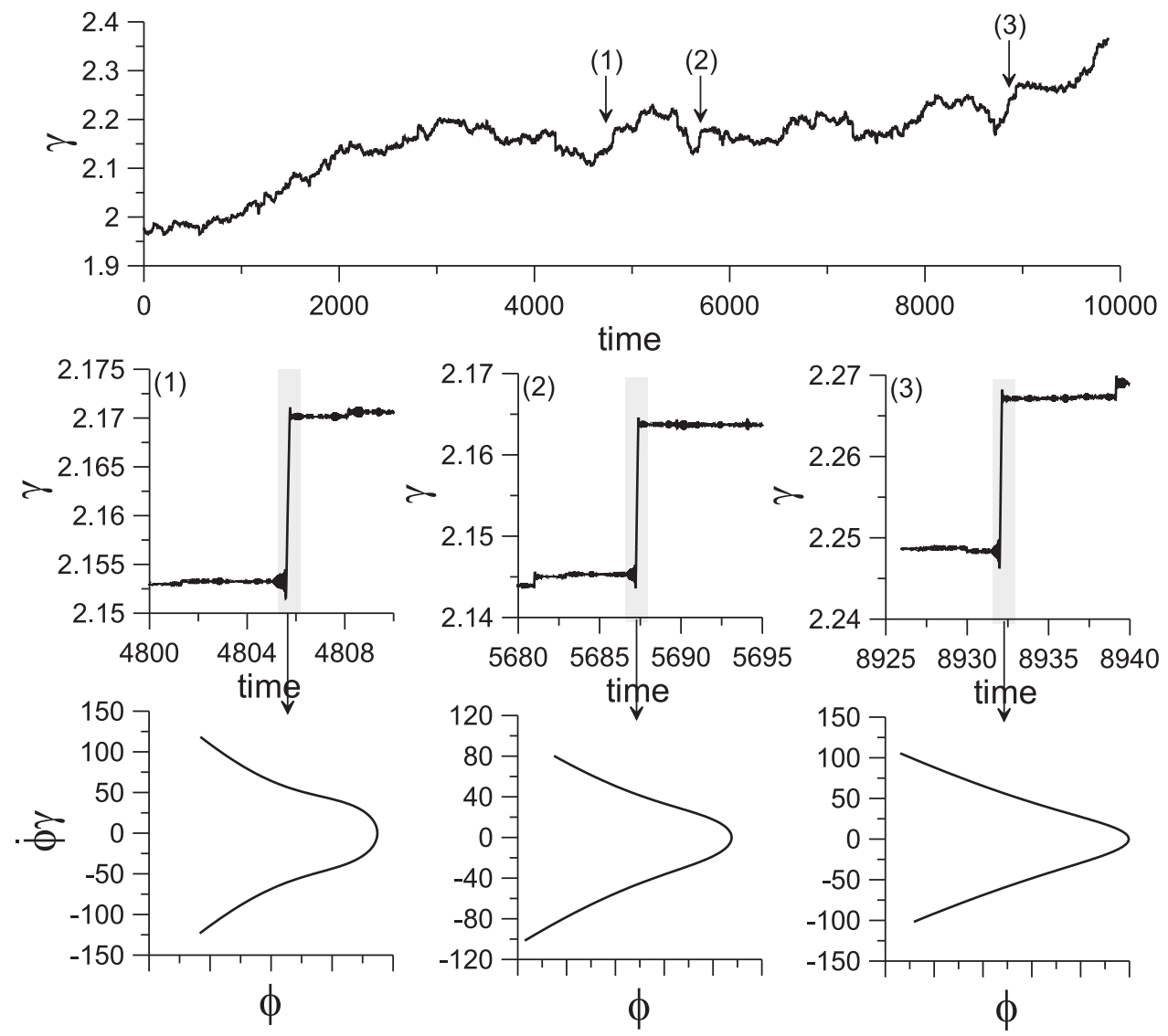

FIG. 8. Evolution of particle energy (the same trajectory is shown as in right bottom panel in Fig. 7) and corresponding fragments of trajectory in $(\phi, \dot{\phi} \gamma)$ plane.

maximizes the effect of reduction of wave-packet width so that the obtained results of modelling (see Fig. 10) represent the absolute minimum of the energy gain. A more moderate reduction of the number of coherent waves would result in larger typical energy gain for a single capture. Fig. 10 shows that even in such unfavourable condition, electrons can gain 10-20 keV for a single trapping-escape event. If we increase the number of periods inside the wave-packets at the equator (one can see in Fig. 1 that one wave-packet can contain more

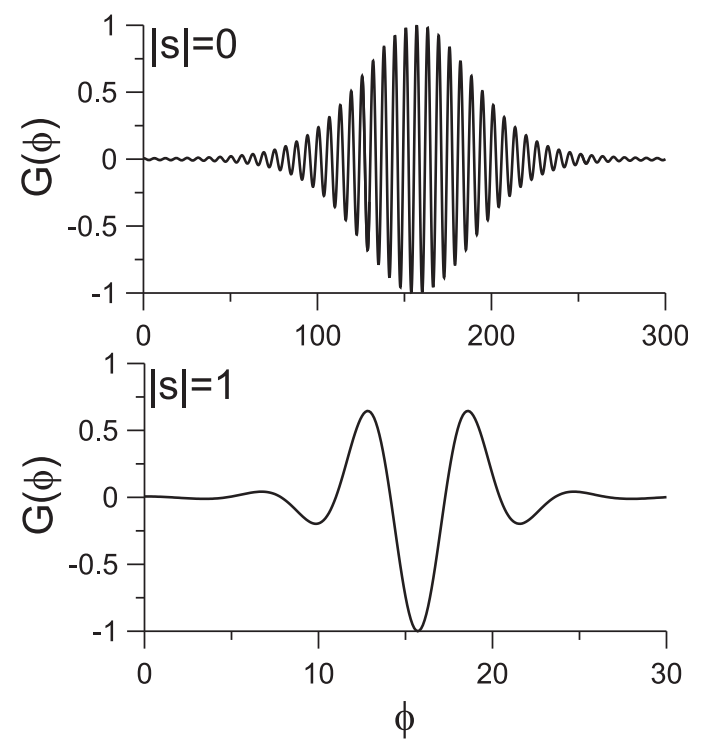

FIG. 9. Wave-packets in the equatorial plane $s=0$ and in the vicinity of reflection point $|s|=1$.

than $10^{3}$ periods), the effect of energy gain reduction would also be weaker.

It is worth mentioning here the effect of a finite value of $k_{\perp}$. Initially, we have a wave with electrostatic potential $\Phi \sim \sin \left(\int k_{\|}\left(s^{\prime}\right) d s^{\prime}-\omega t+k_{\perp} r_{\perp}\right)$, where $r_{\perp}$ is the perpendicular coordinate. We assume that gyrorotation is the fastest motion in the system and corresponding adiabatic invariant $\mu=p_{\perp}^{2} / 2 B_{0} \quad$ is conserved. Then, we can write $r_{\perp}=\sqrt{2 B_{0} \mu} / m_{e} \Omega_{c} \sin \vartheta$, where $\dot{\vartheta}=\Omega_{c}$. We use the following expansion $\sin \left(\int k_{\|}\left(s^{\prime}\right) d s^{\prime}-\omega t+\Lambda \sin \vartheta\right)=\sum J_{n}(\Lambda)$ $\sin \left(\int k_{\|}\left(s^{\prime}\right) d s^{\prime}-\omega t+n \Omega_{c}\right)$, where $J_{n}$ is the Bessel function and $\Lambda=k_{\perp} \sqrt{2 B_{0} \mu} / m_{e} \Omega_{c}$. Because $\Omega_{c} \gg k_{\|} v_{\|}-\omega$ for high latitude with $k_{\|} \ll k_{0}$, we consider Landau resonance $n=0$. In this case, we get a multiplying factor $J_{0}(\Lambda)$ describing the variation of the wave-field in the perpendicular direction. ${ }^{22,37} \mathrm{We}$ do not include this factor in our calculations, because the related decrease of wave amplitude reaches at most a factor $\sim 0.75$. Due to conservation of magnetic moment argument $\Lambda$ varies like $\sim 1 / \sqrt{B_{0}}$ along field line and corresponding variation of $J_{0}(\Lambda)$ could be neglected.

Besides, due to energy conservation, particle acceleration should immediately result in wave damping. This effect does not make it possible to observe large amplitude waves and restricts the efficiency of wave-particle resonant interactions. The solution of this contradiction comes from the impact of transient (non-trapped) particles. These particles transfer their energy to the wave and may compensate damping. ${ }^{44,45}$ As was shown in Ref. 22 (see also review Ref. 2), 

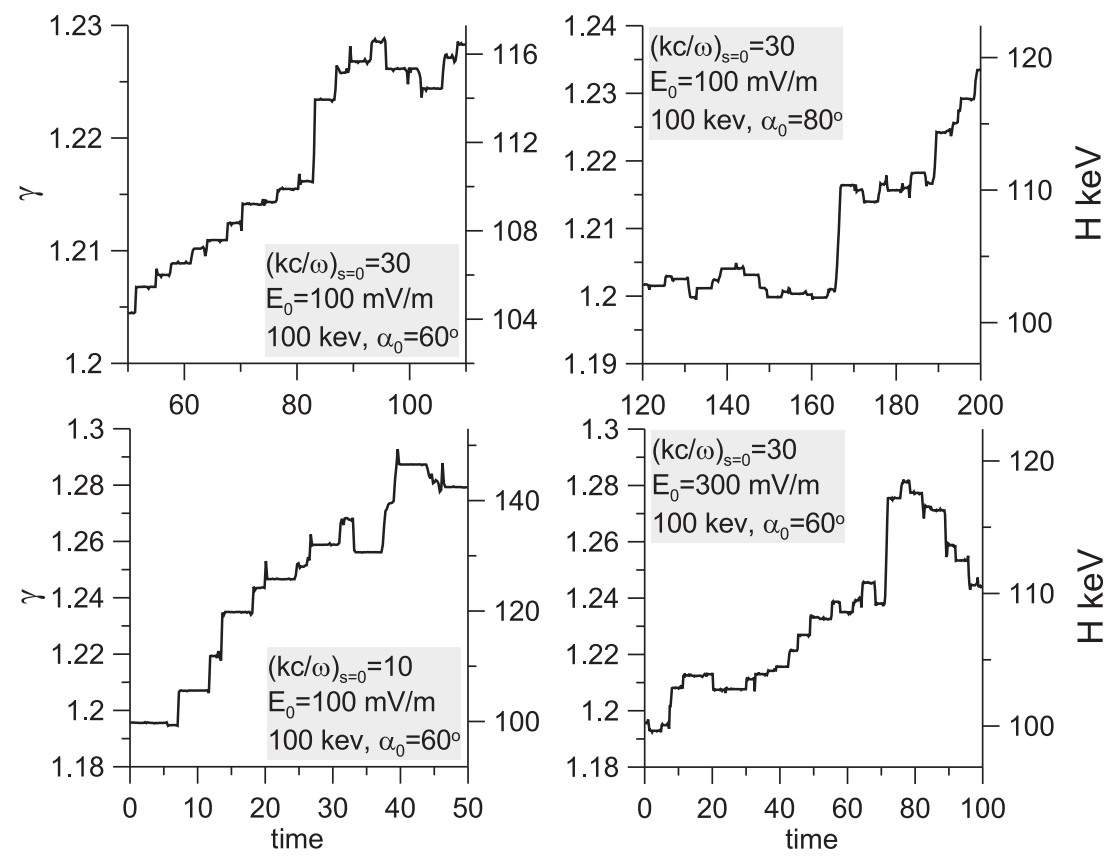

FIG. 10. Jumps of particle energy for various system parameters.

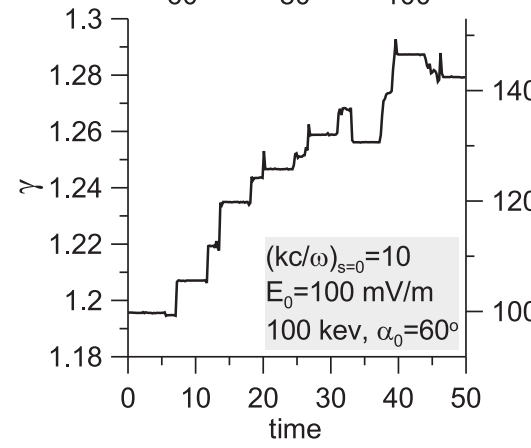

there is no non-diffusive acceleration of the total particle population due to resonant interaction with the wave, while the energy gained by some (trapped) particles is compensated by the energy lost by other (transient) particles. In this case, the wave amplitude can be considered as constant for dynamical equilibrium of the system containing waves and both transient and trapped particles.

\section{DISCUSSIONS AND CONCLUSIONS}

Particle trapping by the wave is a probabilistic process with a certain probability $\mathrm{P}$, see Ref. 40. This probability $\mathrm{P}$ can be found for any particular system as the ratio of the phase flux through the area surrounded by the separatrix (or velocity of evolution of this area) and the total flux through the phase volume $(\phi, \gamma \dot{\phi})$. We derive the expression for $\mathrm{P}$ in Appendix A. It can be shown that $\mathrm{P}>0$ only in the nearequatorial region, where $d \mathrm{~B} / d s>0$ (i.e., at $|s|<s_{0}$ ). Therefore, particles can be captured by waves if their equatorial pitch-angle is larger than $\sim 60^{\circ}$ (for electrons with smaller pitch-angle, the Landau resonance condition $p_{\|} / \gamma \approx v_{R}$ is satisfied at latitudes such that $d \mathrm{~B} / d s<0$ and $\mathrm{P}=0$ ). For particles with initial energy around $100 \mathrm{keV}$, the probability $\mathrm{P}$ varies from $5 \times 10^{-3}$ to $5 \times 10^{-2}$ depending on system parameters (i.e., dimensional time interval between two trappings is $\sim 1 / \mathrm{P} \in[20,200] \mathrm{s})$. Therefore, a corresponding estimate of timescale between susceptive trappings $t_{0} \sim$ $100 \mathrm{~s}$ can be used. This timescale is much smaller than corresponding scales for particle diffusive acceleration evaluated considering moderately oblique whistler waves, ${ }^{6,46}$ and still smaller than characteristic timescales for diffusive acceleration by very oblique whistler waves. ${ }^{47}$

In this paper, we consider mostly strong whistler waves with electric field amplitude $\sim 100 \mathrm{mV} / \mathrm{m}$. Although such waves have been observed in the radiation belts during many spacecraft missions, ${ }^{15-18}$ these observations are relatively rare. Most often, spacecrafts detect wave-forms with amplitudes around tens of $\mathrm{mV} / \mathrm{m}$ (see, e.g., Fig. 1). Such waves cannot substantially accelerate high-energy ( $>100 \mathrm{keV})$ electrons during a single trapping-release event via Landau resonance. Nevertheless, the Landau resonant interaction of waves such that $E_{0} \sim 20-50 \mathrm{mV} / \mathrm{m}$ with low-energy electrons $(\sim 10 \mathrm{keV})$ does result in an effective acceleration, with a typical energy gain for a single trapping event around 10 $50 \mathrm{keV}$ (see Appendix C). It is worth noting that for high pitch-angle electrons such that $\alpha_{0}>60^{\circ}-70^{\circ}$, which usually represent the main part of the trapped population in the Van Allen belts, cyclotron resonance with quasi-parallel whistler waves is generally not available at $L \sim 4$ to 5 for energies $E<60 \mathrm{keV}$ to $100 \mathrm{keV}$ (e.g., see Ref. 20). Therefore, the Landau-resonant acceleration mechanism proposed here should be more efficient for $E \sim 10 \mathrm{keV}$ to $100 \mathrm{keV}$. At larger initial energy $E>100 \mathrm{keV}$, conversely, cyclotron resonant acceleration is very effective at medium latitudes where it can act in succession with Landau resonant acceleration (Landau acceleration occurring when electrons travel from the equator up to their mirror points and cyclotron acceleration when electrons bounce back from their mirror points).

Now, let us discuss the possible consequences of the obtained fast acceleration of particles. It is important to note that a rapid increase of parallel electron energy should result in a decrease of the pitch-angle. However, an important part of the parallel energy gained by the particles during their acceleration is actually rapidly stashed under the form of perpendicular energy, due to adiabatic invariance in an increasing magnetic field. As a result, the parallel component of the particles momentum is sensibly less increased than is total momentum, leading to a smaller decrease of their equatorial pitch-angle $\alpha_{0}$. For $\left(\gamma v_{R}\right)^{2} \ll \gamma^{2}-1$ at the escape point, corresponding typically to high initial energy $>100 \mathrm{keV}$, we get after one trapping-escape event a somewhat reduced equatorial pitch-angle $\alpha_{0}^{\text {new }}>45^{\circ}$ (with $\sin \alpha_{0}^{\text {new }} \sim \sqrt{B_{\text {eq }} / B_{0}\left(s_{\text {esc }}\right)} \sim$ $1 / \sqrt{1+(9 / 2) s_{\text {esc }}^{2}}$ from adiabatic invariance-e.g., see Fig. 5 and also Fig. 6 with $p_{\|}$and energy before trapping and after 
escape). Nevertheless, this moderate reduction of the pitchangle could be somewhat compensated by important quasilinear pitch-angle diffusion provided by oblique whistler waves on the dayside. ${ }^{46,48,49}$ Such diffusion could compensate the decrease of pitch-angle and transfer more parallel energy into perpendicular energy. ${ }^{47}$ For $100 \mathrm{keVs}$ particles and slightly oblique whistler waves, a typical value of the pitch-angle diffusion coefficient is about $10^{-3} \mathrm{~s}^{-1}$, see Ref. 7, but for very oblique waves the diffusion coefficient can be two orders of magnitude larger (see Refs. 48 and 49), comparable to the inverse of a typical trapping-escape period $t_{0} \sim 100 \mathrm{~s}$. Stochastic diffusion tends, in general, to scatter particles toward regions of depleted phase space density. Thus, cyclotron resonant diffusion by whistler waves should mainly scatter small pitch-angle electrons with $\alpha_{0}<45^{\circ}$ toward the loss-cone, while higher pitch-angle electrons with $\alpha_{0}>45^{\circ}$ should mainly get accelerated with the increase of their pitch-angle (e.g., see Ref. 47).

Consequently, for high initial energy $E>100 \mathrm{keV}$ such that $\alpha_{0}^{\text {new }}>45^{\circ}$ (corresponding to rough condition $s_{e s c}$ $\leq 1 / 2$ ), electrons accelerated after one capture-escape event should remain trapped in the belts, resulting in a net energization of the population. They could even get trapped and accelerated several times, due to the compensating effect of quasi-linear pitch-angle diffusion toward $90^{\circ}$. For initial energy around $500 \mathrm{keV}$ or more, the increase of $p_{\|}$after one trapping becomes substantially smaller than the initial value of $p_{\|}$. These particles can really be trapped several times and form a distribution with a power-law tail $\sim \gamma^{\tilde{C}}$. The index $\tilde{C}$ depends on the value of the pitch-angle diffusion coefficient $D_{\alpha \alpha}$ and characteristic time $t_{0}$. For reasonable parameters, we can estimate $\tilde{C}>-2$ (see Appendix B). At such high energies $E>100 \mathrm{keV}$, however, cyclotron resonant trapping and acceleration by quasi-parallel waves should also be considered together with the Landau resonant mechanism for oblique waves, depending on the actual wave-normal angle distribution of the whistler waves.

For low initial energies $E<50 \mathrm{keV}$, we can estimate for Landau resonance alone that trapping-accelerated electrons are such that $\alpha_{0}^{\text {new }}<30^{\circ}$. A very significant decrease of the pitch-angle in the course of one single trapping can then be obtained for high enough wave amplitude. It should result in a rapid increase of the electron flux into the loss-cone, leading to direct precipitation in the atmosphere. A relative isotropization of the electron distribution could also be observed. The trapping of low energy electrons $<50 \mathrm{keV}$ by quasi-electrostatic whistler waves could, therefore, explain the microburst precipitations of electrons in the range 20$200 \mathrm{keV}$ observed on the dayside in correlation with intense whistler emissions (see, e.g., Refs. 50-54).

We would like to mention also one more application of the obtained results alternative to radiation belt physics. In the Earth magnetotail, fast nonlinear waves propagating from the deep tail to the Earth are often observed (so-called dipolarization fronts, see, e.g., Refs. 55 and 56). These structures are associated with electron perpendicular heating ${ }^{57,58}$ and the corresponding generation of whistler waves with large amplitude. ${ }^{59}$ Electron trapped and accelerated by these waves could be responsible, as we showed above, for scatter- ing into the loss-cone and corresponding precipitations. Indeed, observations of dipolarization fronts are often correlated with such precipitations (see, e.g., Refs. 60 and 61).

In conclusion, we have considered a Landau resonant non-diffusive mechanism of electron interaction with large amplitude whistler waves. We have demonstrated that electron trapping can result in an energy gain of up to $100 \mathrm{keV}$. For electrons with initial energy below $100 \mathrm{keV}$, such an acceleration corresponds to a significant decrease in pitchangle and a subsequent scattering into the loss-cone. Conversely, electrons with initial energy of $200 \mathrm{keV}$ to $500 \mathrm{keV}$ can significantly increase their energy due to several successive interactions with the waves. If one takes into account additional cyclotron quasi-linear pitch-angle diffusion, then these accelerations could result in the formation of a powerlaw energy distribution.

This work was supported by CNES through the grant "Modele d'ondes." Work of A.O. was supported by the STUDIUM program of the Region Centre in France. Authors thank the ESA Cluster Active Archive for providing the STAFF-SA data set.

\section{APPENDIX A: PROBABILITY OF TRAPPING}

Trapping of particles into the resonance is a probabilistic process $^{40}$ with a certain probability P. To define P, we plot the scheme of the separatrix at two successive moments of time in Fig. 11. If the area surrounded by the separatrix $S$ grows (i.e., moment $t^{*}$ precedes moment $t^{* *}$ in scheme Fig. 11), then new particles from the outer region can become trapped by the wave. We obtain an expression for $\mathrm{P}$ in case of nonrelativistic particles, when Hamiltonian (4) can be written as

$$
\left\{\begin{array}{c}
H_{\phi}=\frac{1}{2} \dot{\phi}^{2}+\kappa(\mathrm{A}(s) \phi+\mathrm{B}(s) \sin \phi) \\
\mathrm{A}(s)=\frac{\omega^{2}}{\kappa^{2}}(1-s)^{-2}+\frac{9}{2} \Omega_{b}^{2} s(1-s) \\
\mathrm{B}(s)=\varepsilon(1-s)^{2},
\end{array}\right.
$$

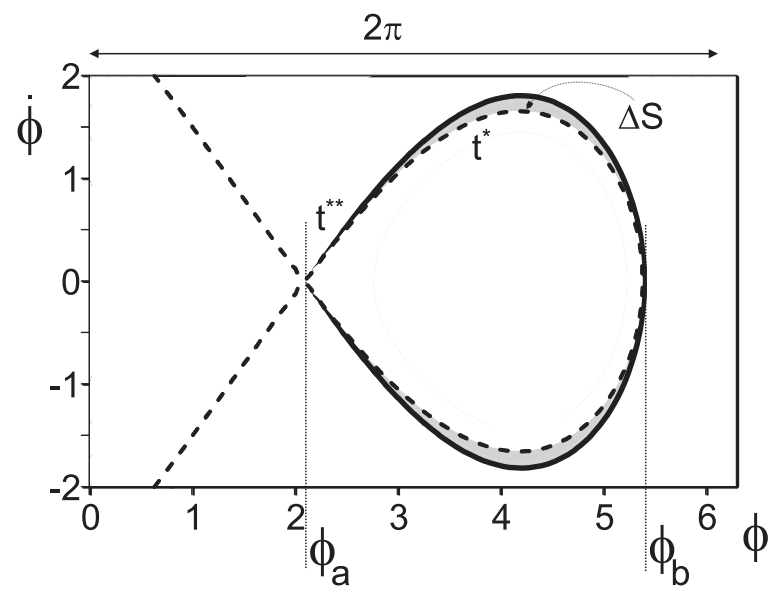

FIG. 11. Schematic view of the separatrix for two moments $t^{*}, t^{* *}$. Grey color is used for the difference area surrounded by the separatrices. 
where for simplicity only $s>0$ half-space is considered. Expression for area $S=\oint \dot{\phi} d \phi$ has a form

$$
\begin{gathered}
S=\oint \sqrt{2 H_{\phi}-\kappa(\mathrm{A}(s) \phi+\mathrm{B}(s) \sin \phi)} d \phi=2 \kappa^{1 / 2} \times \\
\int_{\phi_{a}}^{\phi_{b}} \sqrt{\left(\mathrm{A}(s) \phi_{a}+\mathrm{B}(s) \sin \phi_{a}\right)-(\mathrm{A}(s) \phi+\mathrm{B}(s) \sin \phi)} d \phi
\end{gathered}
$$

where $\phi_{a}$ and $\phi_{b}$ are indicated in Fig. 11. The change of $S$ with time is characterized by the expression

$$
\dot{S}=\frac{d S}{d t}=\frac{\partial S}{\partial s} \dot{s}=\frac{\partial S}{\partial s} v_{R}
$$

We plot $S$ as function of $s$ for various system parameters in Fig. 12. One can see that for range $s>s_{0}$, area $S$ decreases with $s$ and, as a result, $\dot{S}<0$ (i.e., moment $t^{* *}$ precedes the moment $t^{*}$ in the scheme of Fig. 11). Therefore, a particle cannot become trapped in this region with $\dot{s}=p_{\|} / \gamma>0$. Trapping in $s \in\left[s_{0}, 1\right]$ corresponds to the backward motion of the particle (i.e., $\dot{s}<0$ ) with energy decrease.

For range $s \in\left[0, s_{0}\right]$, area $S$ increases with $s$ and we have $\dot{S}>0$. (i.e., moment $t^{*}$ precedes the moment $t^{* *}$ in scheme Fig. 11 and trapping of new particles is possible). Therefore, we can define the probability of particle trapping in each point in the range $s \in\left[0, s_{0}\right]$. To obtain an expression of probability $\mathrm{P}$, we need to calculate the whole flux of phase volume $\Upsilon=d V / d t$ (where phase volume is $V=\int \dot{\phi} d \phi$ ) and compare it with $\dot{S}$. Expression for $\Upsilon$ is
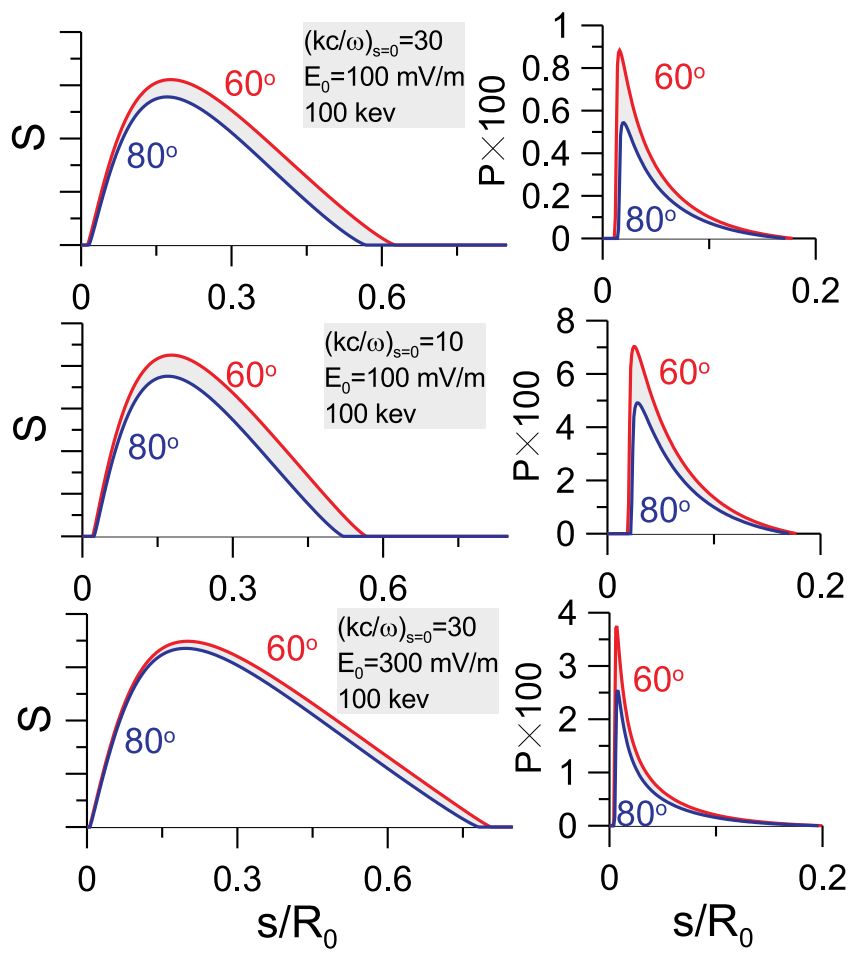

FIG. 12. Area under separatrix $S$ as function of $s$ for various system parameters and two values of $\alpha_{0}$. Right panel shows dependence of probability $\mathrm{P}$ on $s$.
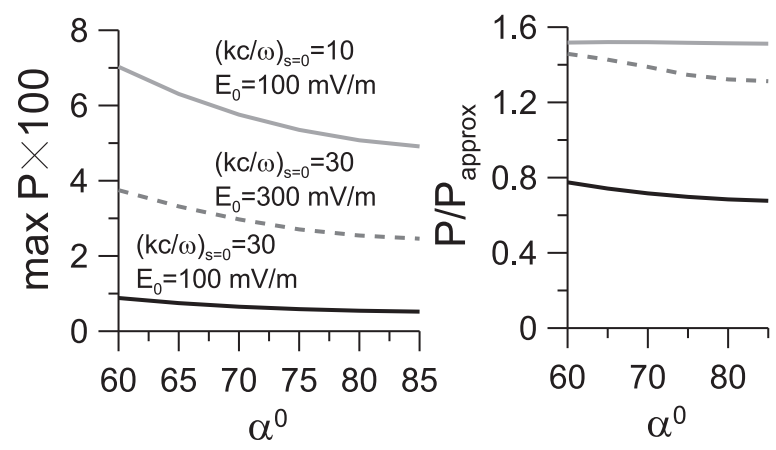

FIG. 13. Maximum value of probability as function of equatorial pitch-angle $\alpha_{0}$ for initial energy $100 \mathrm{keV}$. Right panel shows ratio of maxP and approximated value of probability $\mathrm{P}_{\text {approx }}$.

$$
\Upsilon=\int_{0}^{2 \pi} \ddot{\phi} d \phi=\kappa \int_{0}^{2 \pi}(\mathrm{A}(s)+\mathrm{B}(s) \cos \phi) d \phi=2 \pi \kappa \mathrm{A}(s) .
$$

Probability $\mathrm{P}=\dot{S} / \Upsilon$ as function of $s$ is shown in Fig. 12 . Probability increases with the wave amplitude $E_{0}$ and $\mathrm{P} \sim 1 / \sqrt{\kappa}$. Particles with smaller equatorial pitch-angle $\alpha_{0}$ have a larger probability to become trapped.

Function $\mathrm{P}(s)$ has local maximum corresponding to the maximum value of $d S / d s$ and we can define the value of maxP. Dependence of maxP on initial pitch-angle $\alpha_{0}$ is shown in Fig. 13 (left panel). The magnitude of probability is about $\operatorname{maxP} \sim 10^{-2}$ and typical electrons can become trapped after 100 bounce oscillations (or after $10 \mathrm{~s}$ ). It means that for an ensemble of particles with energy around $100 \mathrm{keV}$ and pitch-angles with the range $\alpha_{0} \in\left[60^{\circ}, 80^{\circ}\right]$, every hundredth particle become trapped during one single bounce period.

Taking into account the profile of $S(s)$, one can obtain an approximated expression for probability P. To this end, one can approximate the smooth growth of $S$ with $s$ by stepwise function $\hat{S} \approx S(s) \Theta\left(|s| / s_{0}-1\right)$. In this case, the calculation of the derivative $\dot{S}$ gives

$$
\dot{S}=\frac{\partial \hat{S}}{\partial s} \dot{S}=S \delta\left(|s| / s_{0}-1\right)\left(v_{R} / s_{0}\right)+\frac{\partial S}{\partial s} \Theta\left(|s| / s_{0}-1\right) v_{R} .
$$

In the point $|s|=s_{0}$, the second term can be neglected and $\dot{S}=S\left(s_{0}\right)\left(v_{R} / s_{0}\right)$. The corresponding expression for probability is $\mathrm{P}_{\text {approx }}=S\left(s_{0}\right) v_{R} / 2 \pi \kappa s_{0} A\left(s_{0}\right)$. We plot ratio $\operatorname{maxP} / \mathrm{P}_{\text {approx }}$ in the right panel in Fig. 13. One can see that our approximate expression is correct within the factor of $\sim 2$.

\section{APPENDIX B: FORMATION OF THE ENERGY DISTRIBUTION}

In this appendix, we consider formation of the energy distribution due to acceleration of trapped particles in the case of small pitch-angle change $\Delta \alpha$ for one trapped-escape event (i.e., for relatively large initial energy). We take initial distribution as $f_{0}=N_{0} \delta\left(\alpha-\alpha_{0}\right) \delta\left(\gamma-\gamma_{0}\right)$, where $N_{0}$ is the initial phase density of particles with energy $\gamma \in\left[\gamma_{0}-\delta \gamma\right.$, $\left.\gamma_{0}+\delta \gamma\right]$ and pitch-angle $\alpha \in\left[\alpha_{0}-\delta \alpha, \alpha_{0}+\delta \alpha\right]$ (here, we 

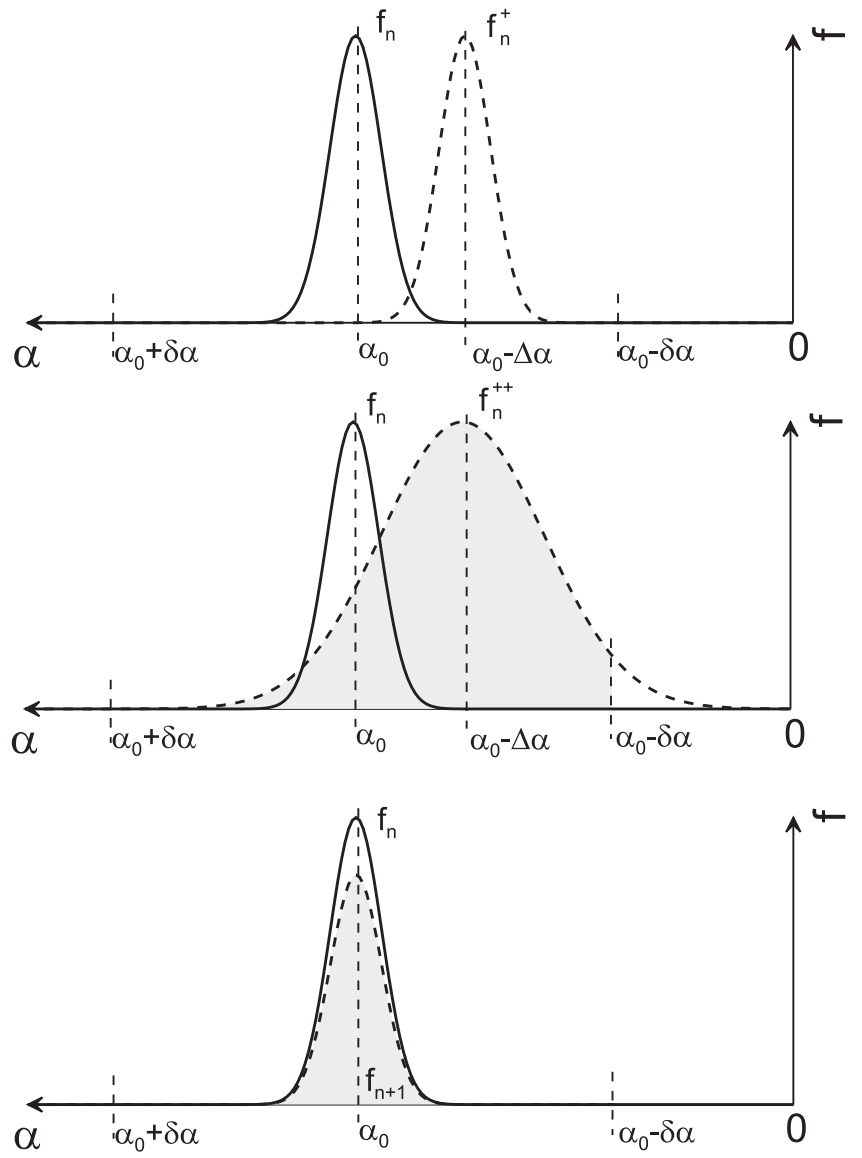

FIG. 14. Schematic view of evolution of velocity distribution for three moments. Grey color of $f_{n+1}$ distribution shows phase density which returns into the region of resonant interaction $\alpha \in\left[\alpha_{0}-\delta \alpha, \alpha_{0}+\delta \alpha\right]$.

assume that $\delta \alpha \ll \alpha_{0}$ and $\left.\delta \gamma \ll \gamma_{0}\right)$. Then, the first resonant jump of energy results in the formation of a new distribution $f_{0}^{+}=N_{0} \delta\left(\alpha-\alpha_{0}+\Delta \alpha\right) \delta\left(\gamma-\gamma_{0}-\Delta \gamma\right)$. This distribution should be used as an initial condition for pitch-angle diffusion equation. ${ }^{62} \mathrm{We}$ expand this equation around $\alpha_{0}$ to obtain

$$
\frac{\partial f}{\partial t}=D_{0} \frac{\partial^{2} f}{\partial \alpha^{2}}
$$

where $D_{0}=D_{\alpha \alpha}\left(\alpha_{0}, \gamma_{0}\right)=$ const. Solution of this equation is

$$
f_{0}^{++}=\frac{N_{0} \delta\left(\gamma-\gamma_{0}-\Delta \gamma\right)}{\sqrt{4 \pi D_{0} t}} \exp \left(-\frac{\left(\alpha-\alpha_{0}+\Delta \alpha_{0}\right)^{2}}{4 D_{0} t}\right) .
$$

Then, after the time interval $t_{0} \sim 1 / \mathrm{P}$ (interval between successive resonant energy gains; we also assume for simplicity that $t_{0}=$ const and does not depend on energy or pitchangle), we can calculate phase density in the interval of the resonant interaction $\alpha \in\left[\alpha_{0}-\delta \alpha, \alpha_{0}+\delta \alpha\right]$ as

$$
\begin{aligned}
N_{1} & =N_{0} \int_{\alpha_{0}-\delta \alpha}^{\alpha_{0}+\delta \alpha} f_{0}^{++} d \alpha \approx N_{0}\left(1-\int_{\alpha_{0}-\delta \alpha}^{\alpha_{0}+\delta \alpha-\Delta \alpha} f_{0}^{++} d \alpha\right) \\
& =N_{0}\left(1-\frac{1}{2}\left(\operatorname{erf}\left(x_{+}\right)-\operatorname{erf}\left(x_{-}\right)\right)\right) \delta\left(\gamma-\gamma_{0}-\Delta \gamma\right),
\end{aligned}
$$

where

$$
\begin{aligned}
& x_{+}=\delta \alpha / \sqrt{4 D_{0} t_{0}} \\
& x_{-}=(\delta \alpha-\Delta \alpha) / \sqrt{4 D_{0} t_{0}}
\end{aligned}
$$

Now, we can write distribution function as $f_{1}=N_{1} \delta\left(\alpha-\alpha_{0}\right) \delta\left(\gamma-\gamma_{0}-\Delta \gamma\right)$. Next resonant interaction gives us $f_{1}^{+}=N_{1} \delta\left(\alpha-\alpha_{0}+\Delta \alpha\right) \delta\left(\gamma-\gamma_{0}-2 \Delta \gamma\right)$ and so on (see scheme in Fig. 14).

Thus, for moment $n+1$, we have phase densities $N_{n+1}=C N_{n}$ of particles with energy $\gamma_{n+1}=\gamma_{0}+n \Delta \gamma$, where $C$ is

$$
\begin{aligned}
C & =1-\frac{1}{2}\left(\operatorname{erf}\left(x_{+}\right)-\operatorname{erf}\left(x_{-}\right)\right) \\
& \approx 1-\frac{1}{\sqrt{\pi}} \exp \left(-\frac{(\delta \alpha)^{2}}{4 D_{0} t_{0}}\right) \frac{\Delta \alpha}{\sqrt{4 D_{0} t_{0}}}=1+\tilde{C}(\Delta \gamma / \gamma) .
\end{aligned}
$$

Here, we expand $C$ around $\Delta \alpha \approx \alpha_{0}(\Delta \gamma / \gamma)$ and introduce constant $\tilde{C}$ depending on $D_{0}, t_{0}, \alpha_{0}$, and $\delta \alpha$. Then, we can write $\Delta N=N_{n+1}-N_{n}=\underset{\widetilde{C}}{\tilde{C}} N_{n}(\Delta \gamma / \gamma)$. Integration of this equation gives $N(\gamma) \sim \gamma \tilde{C}$ with $\tilde{C}=-\left(\alpha_{0} / \sqrt{\pi} \delta \alpha\right) R(d)$,
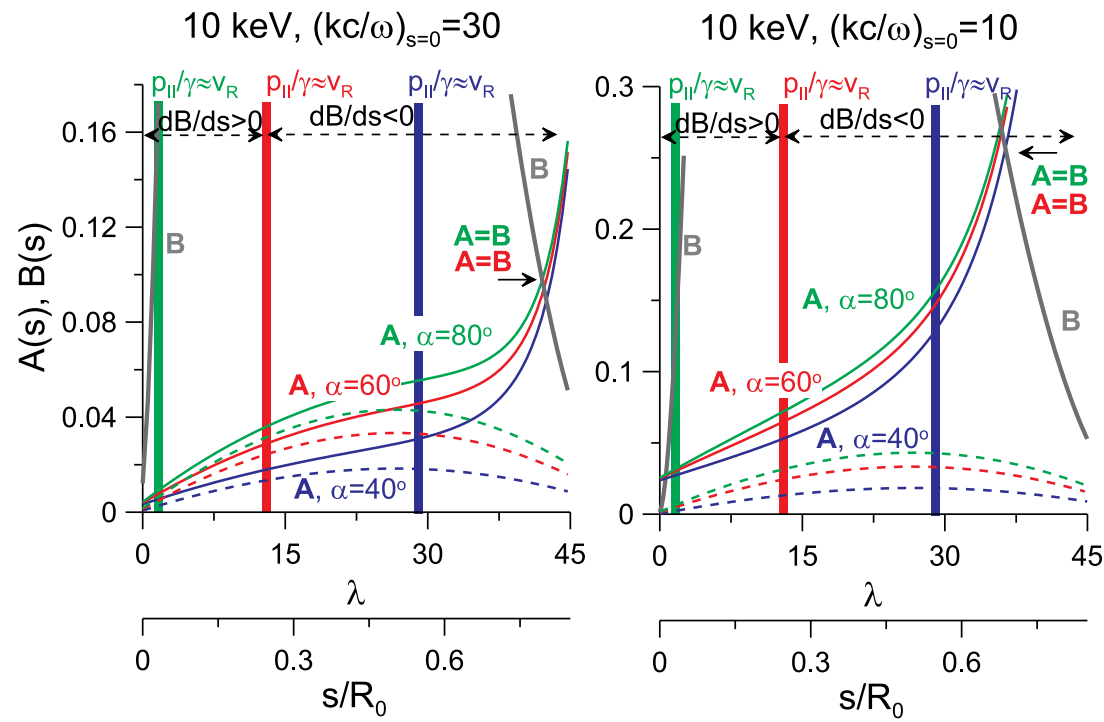

FIG. 15. The same as in Fig. 5, but for $10 \mathrm{keV}$. 

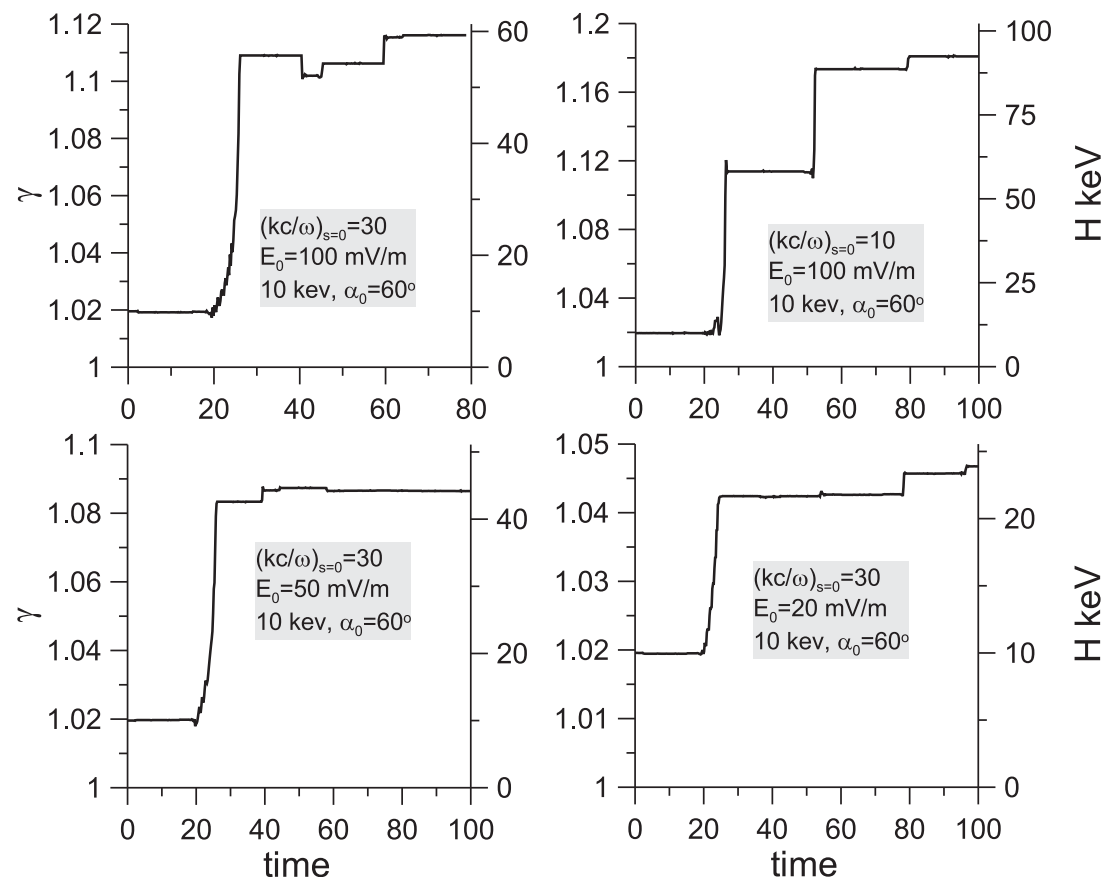

FIG. 16. Jumps of particle energy for various system parameters and low initial energy.

where $R(d)=d \exp \left(-d^{2}\right)$ and $d=\delta \alpha / \sqrt{4 D_{0} t_{0}}$. Function $R(d)$ varies from 0 up to $2^{-1 / 2} \exp (-1 / 2) \approx 0.43$. Therefore, for $\alpha_{0} \sim 60-80^{\circ}$ and $\delta \alpha \sim 10^{\circ}$, we estimate $\tilde{C}>-2$. One can see that degree $\tilde{C}$ strongly depends on diffusion rates $D_{0}$ and time interval $t_{0}$.

\section{APPENDIX C: DYNAMICS OF SMALL ENERGY PARTICLES}

In this appendix, we consider the acceleration of electrons with energy $\sim 10 \mathrm{keV}$. In contract to high energy electrons, for which we can neglect the effect of $v_{R}(s)$ variation with $s$ for estimation of energy gain, here we need to take into account the inhomogeneity of the phase velocity. Fig. 15 shows coefficients $\mathrm{A}$ and $\mathrm{B}$ for system with $10 \mathrm{keV}$ particles and two value of $(k c / \omega)_{s=0}$. Comparison of Fig. 15 and Fig. 5 demonstrates the main difference between small energy and high energy particles: for $10 \mathrm{keV}$ electrons, the position of escape from the resonance $s_{\text {esc }}$ is defined by the first term of A corresponding to $v_{R}$ inhomogeneity, i.e., by $\sim(\omega \gamma / \kappa)^{2}(1-s)^{-2}$. This effect can be explained by the decrease of $\Omega_{b}^{2} \sim \gamma_{0}^{2}-1$ with the decrease of $\gamma_{0}$. Therefore, for small energy, the electron point of escape $s_{\text {esc }}$ can be found as

$$
s_{\text {esc }}=1-\left(\omega^{2} \gamma_{0} / \kappa^{2} \varepsilon\right)^{1 / 4}
$$

where we assume $\eta\left(s_{\text {esc }}\right) \approx 1$. The corresponding energy gain for $\gamma \approx \gamma_{R} \approx 1+v_{R}^{2} / 2$ (due to $\Omega_{b}^{2} \ll 1$ and $v_{R} \ll 1$ ) is

$$
\begin{aligned}
\Delta \gamma_{\max } & \approx \frac{\partial \gamma_{R}}{\partial s} s_{e s c} \approx \frac{s_{e s c}}{2} \frac{\partial v_{R}^{2}}{\partial s} \approx \frac{\omega^{2}}{\kappa^{2}} \frac{s_{e s c}}{\left(1-s_{e s c}\right)^{3}} \\
& \approx \varepsilon s_{e s c}\left(1-s_{e s c}\right)<\frac{\varepsilon^{3 / 4} \omega^{1 / 2}}{\kappa^{1 / 2}} .
\end{aligned}
$$

This estimate gives energy gains up to hundred keVs, depending on system parameters. Several examples of parti- cle acceleration due to trapping by waves with various amplitudes are shown in Fig. 16. One can see that energy gain is about $20-50 \mathrm{keV}$ and depends on $E_{0}$ and $(k c / \omega)_{s=0}$. This gain is much larger than the initial particle energy. As a result, trapping and acceleration of $\sim 10 \mathrm{keV}$ electrons should result in effective particle precipitation into the loss-cone due to the more rapid decrease of their pitch-angle than for higher energy electrons.

${ }^{1}$ Y. Y. Shprits, D. A. Subbotin, N. P. Meredith, and S. R. Elkington, J. Atmos. Sol.-Terr. Phys. 70, 1694-1713 (2008).

${ }^{2}$ D. Shklyar and H. Matsumoto, Surv. Geophys. 30, 55-104 (2009).

${ }^{3}$ R. M. Thorne, B. Ni, X. Tao, R. B. Horne, and N. P. Meredith, Nature (London) 467, 943-946 (2010)

${ }^{4}$ D. Summers, R. M. Thorne, and F. Xiao, J. Geophys. Res. 103, $20487-$ 20500, doi:10.1029/98JA01740 (1998)

${ }^{5}$ Y. Katoh and Y. Omura, J. Geophys. Res. 109, A12214, doi:10.1029/ 2004JA010654 (2004).

${ }^{6}$ R. B. Horne, R. M. Thorne, S. A. Glauert, J. M. Albert, N. P. Meredith, and R. R. Anderson, J. Geophys. Res. 110, A03225, doi:10.1029/ 2004JA010811 (2005).

${ }^{7}$ B. Ni, R. M. Thorne, N. P. Meredith, Y. Y. Shprits, and R. B. Horne, J. Geophys. Res. 116, A10207, doi:10.1029/2011JA016517 (2011).

${ }^{8}$ V. I. Karpman and D. R. Shkliar, Planet. Space Sci. 25, 395-403 (1977).

${ }^{9}$ V. Y. Trakhtengerts, Ann. Geophys. 17, 95-100 (1999).

${ }^{10}$ A. G. Demekhov, V. Y. Trakhtengerts, M. J. Rycroft, and D. Nunn, Geomagn. Aeron. 46, 711-716, doi:10.1134/S0016793206060053 (2006).

${ }^{11}$ Y. Omura, N. Furuya, and D. Summers, J. Geophys. Res. 112, A06236, doi:10.1029/2006JA012243 (2007)

${ }^{12}$ D. Nunn, J. Plasma Phys. 6, 291 (1971).

${ }^{13}$ V. I. Karpman, J. N. Istomin, and D. R. Shklyar, Plasma Phys. 16, 685703 (1974)

${ }^{14}$ T. F. Bell, J. Geophys. Res. 91, 4365-4379, doi:10.1029/ JA091iA04p04365 (1986).

${ }^{15}$ C. M. Cully, J. M. Bonnell, and R. E. Ergun, Geophys. Res. Lett. 35, L17S16, doi:10.1029/2008GL033643 (2008).

${ }^{16}$ C. Cattell, J. R. Wygant, K. Goetz, K. Kersten, P. J. Kellogg, T. von Rosenvinge, S. Bale, I. Roth, M. Temerin, M. K. Hudson, R. A. Mewaldt, M. Wiedenbeck, M. Maksimovic, R. Ergun, M. Acuna, and C. T. Russell, Geophys. Res. Lett. 35, L01105, doi:10.1029/2007GL032009 (2008).

${ }^{17}$ P. J. Kellogg, C. A. Cattell, K. Goetz, S. J. Monson, and L. B. Wilson III, J. Geophys. Res. 116, A09224, doi:10.1029/2010JA015919 (2011). 
${ }^{18}$ L. B. Wilson, C. A. Cattell, P. J. Kellogg, J. R. Wygant, K. Goetz, A. Breneman, and K. Kersten, Geophys. Res. Lett. 38, L17107, doi:10.1029/ 2011GL048671 (2011).

${ }^{19}$ I. Roth, M. Temerin, and M. K. Hudson, Ann. Geophys. 17, 631-638 (1999).

${ }^{20}$ J. Bortnik, R. M. Thorne, and U. S. Inan, Geophys. Res. Lett. 35, L21102, doi:10.1029/2008GL035500 (2008).

${ }^{21}$ P. H. Yoon, Geophys. Res. Lett. 38, L12105, doi:10.1029/2011GL047893 (2011).

${ }^{22}$ V. V. Solovev and D. R. Shkliar, Sov. Phys. JETP 63, 272-277 (1986).

${ }^{23}$ D. R. Shklyar and F. Jiříček, J. Atmos. Sol.-Terr. Phys. 62, 347-370 (2000).

${ }^{24}$ J. Bortnik, R. M. Thorne, and N. P. Meredith, Nature (London) 452, 62-66 (2008).

${ }^{25}$ H. Breuillard, Y. Zaliznyak, V. Krasnoselskikh, O. Agapitov, A. Artemyev, and G. Rolland, Ann. Geophys. 30, 1223-1233 (2012).

${ }^{26}$ N. Haque, M. Spasojevic, O. Santolík, and U. S. Inan, J. Geophys. Res. 115, A00F07, doi:10.1029/2009JA014717 (2010).

${ }^{27}$ O. Agapitov, V. Krasnoselskikh, Y. V. Khotyaintsev, and G. Rolland, Geophys. Res. Lett. 382, L20103, doi:10.1029/2011GL049597 (2011).

${ }^{28} \mathrm{~W}$. Li, J. Bortnik, R. M. Thorne, and V. Angelopoulos, J. Geophys. Res. 116, A12205, doi:10.1029/2011JA017035 (2011).

${ }^{29}$ V. L. Ginzburg and A. Rukhadze, Waves in Magnetoactive Plasma, 2nd ed. (Nauka, Moscow, 1975).

${ }^{30}$ S. S. Sazhin and R. B. Horne, Planet. Space Sci. 38, 1551-1553 (1990).

${ }^{31}$ B. T. Tsurutani, B. J. Falkowski, O. P. Verkhoglyadova, J. S. Pickett, O. Santolík, and G. S. Lakhina, J. Geophys. Res. 116, A09210, doi:10.1029/ 2010JA016237 (2011)

${ }^{32}$ D. A. Gurnett, R. L. Huff, J. S. Pickett, A. M. Persoon, R. L. Mutel, I. W. Christopher, C. A. Kletzing, U. S. Inan, W. L. Martin, J.-L. Bougeret, H. S. C. Alleyne, and K. H. Yearby, Ann. Geophys. 19, 1259-1272 (2001).

${ }^{33}$ N. Cornilleau-Wehrlin, G. Chanteur, S. Perraut, L. Rezeau, P. Robert, A. Roux, C. de Villedary, P. Canu, M. Maksimovic, Y. de Conchy, D. H. C. Lacombe, F. Lefeuvre, M. Parrot, J. L. Pinçon, P. M. E. Décréau, C. C. Harvey, P. Louarn, O. Santolik, H. S. C. Alleyne, M. Roth, T. Chust, O. Le Contel, and Staff Team, Ann. Geophys. 21, 437-456 (2003).

${ }^{34}$ R. E. Denton, K. Takahashi, I. A. Galkin, P. A. Nsumei, X. Huang, B. W. Reinisch, R. R. Anderson, M. K. Sleeper, and W. J. Hughes, J. Geophys. Res. 111, A04213, doi:10.1029/2005JA011414 (2006).

${ }^{35}$ N. P. Meredith, R. B. Horne, R. M. Thorne, and R. R. Anderson, Geophys. Res. Lett. 30, 1871, doi:10.1029/2003GL017698 (2003).

${ }^{36}$ J. R. Cary and A. J. Brizard, Rev. Mod. Phys. 81, 693-738 (2009).

${ }^{37}$ D. R. Shklyar, Sov. Phys. JETP 53, 1197 (1981).

${ }^{38}$ Y. Katoh, Y. Omura, and D. Summers, Ann. Geophys. 26, 3451-3456 (2008).

${ }^{39}$ V. I. Karpman, J. N. Istomin, and D. R. Shklyar, Phys. Lett. A 53, 101102 (1975).

${ }^{40}$ V. I. Arnold, V. V. Kozlov, and A. I. Neishtadt, Mathematical Aspects of Classical and Celestial Mechanics, 3rd ed., Dynamical Systems III. Encyclopedia of Mathematical Sciences (Springer-Verlag, New York, 2006).
${ }^{41}$ A. V. Gurevich and L. P. Pitaevskii, Reviews of Plasma Physics, edited by A. M. A. Leontovich (1986), Vol. 10, p. 1.

${ }^{42}$ J. M. Albert, Phys. Fluids B 5, 2744-2750 (1993).

${ }^{43}$ O. Agapitov, V. Krasnoselskikh, T. D. de Wit, Y. Khotyaintsev, J. S. Pickett, O. Santolík, and G. Rolland, J. Geophys. Res. 116, A09222, doi:10.1029/2011JA016540 (2011).

${ }^{44}$ D. R. Shklyar, Phys. Lett. A 375, 1583-1587 (2011).

${ }^{45}$ D. R. Shklyar, Ann. Geophys. 29, 1179-1188 (2011).

${ }^{46}$ Y. Y. Shprits and B. Ni, J. Geophys. Res. 114, A11205, doi:10.1029/ 2009JA014223 (2009).

${ }^{47}$ D. Mourenas, A. V. Artemyev, J.-F. Ripoll, O. V. Agapitov, and V. V. Krasnoselskikh, J. Geophys. Res. 117, A06234, doi:10.1029/2012JA017717 (2012).

${ }^{48}$ A. Artemyev, O. Agapitov, H. Breuillard, V. Krasnoselskikh, and G. Rolland, Geophys. Res. Lett. 39, L08105, doi:10.1029/2012GL051393 (2012).

${ }^{49}$ D. Mourenas, A. V. Artemyev, J.-F. Ripoll, O. V. Agapitov, and V. V. Krasnoselskikh, J. Geophys. Res. 117, A06234, doi:10.1029/ 2012JA017717 (2012).

${ }^{50}$ M. N. Oliven, D. A. Gurnett, J. Geophys. Res. 73, 2355-2362, doi:10.1029/JA073i007p02355 (1968).

${ }^{51}$ S. Datta, R. M. Skoug, M. P. McCarthy, and G. K. Parks, Geophys. Res. Lett. 23, 1729-1732, doi:10.1029/96GL01672 (1996).

${ }^{52}$ R. M. Thorne, T. P. O'Brien, Y. Y. Shprits, D. Summers, and R. B. Horne, J. Geophys. Res. 110, A09202, doi:10.1029/2004JA010882 (2005).

${ }^{53}$ K. Kersten, C. A. Cattell, A. Breneman, K. Goetz, P. J. Kellogg, J. R. Wygant, L. B. Wilson III, J. B. Blake, M. D. Looper, and I. Roth, Geophys. Res. Lett. 38, 8107, doi:10.1029/2011GL046810 (2011); e-print arXiv:1101.3345 [physics.space-ph].

${ }^{54}$ P. J. Kellogg, C. A. Cattell, K. Goetz, S. J. Monson, and L. B. Wilson III, Geophys. Res. Lett. 37, L20106, doi:10.1029/2010GL044845 (2010).

${ }^{55}$ R. Nakamura, A. Retinò, W. Baumjohann, M. Volwerk, N. Erkaev, B. Klecker, E. A. Lucek, I. Dandouras, M. André, and Y. Khotyaintsev, Ann. Geophys. 27, 1743-1754 (2009).

${ }^{56}$ A. Runov, V. Angelopoulos, M. I. Sitnov, V. A. Sergeev, J. Bonnell, J. P. McFadden, D. Larson, K. Glassmeier, and U. Auster, Geophys. Res. Lett. 36, L14106, doi:10.1029/2009GL038980 (2009).

${ }^{57}$ H. S. Fu, Y. V. Khotyaintsev, M. André, and A. Vaivads, Geophys. Res. Lett. 381, L16104, doi:10.1029/2011GL048528 (2011).

${ }^{58}$ J. Birn, A. V. Artemyev, D. N. Baker, M. Echim, M. Hoshino, and L. M. Zelenyi, Space Sci. Rev. (in press).

${ }^{59}$ Y. V. Khotyaintsev, C. M. Cully, A. Vaivads, M. André, and C. J. Owen, Phys. Rev. Lett. 106, 165001 (2011).

${ }^{60}$ A. Runov, V. Angelopoulos, M. Sitnov, V. A. Sergeev, R. Nakamura, Y. Nishimura, H. U. Frey, J. P. McFadden, D. Larson, J. Bonnell, K. Glassmeier, U. Auster, M. Connors, C. T. Russell, and H. J. Singer, Planet. Space Sci. 59, 517-525 (2011)

${ }^{61}$ L. R. Lyons, Y. Nishimura, X. Xing, A. Runov, V. Angelopoulos, E. Donovan, and T. Kikuchi, J. Geophys. Res. 117, A02212, doi:10.1029/ 2011JA017265 (2012).

${ }^{62}$ Quantitative Aspects of Magnetospheric Physics, edited by L. R. Lyons and D. J. Williams (Reidel, Dordrecht, 1984), p. 15 and 231. 